\title{
Convolutional neural network-based ensemble methods to recognize Bangla handwritten character
}

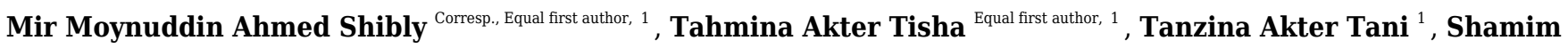 \\ Ripon ${ }^{\text {Corresp. } 1}$ \\ ${ }^{1}$ Department of Computer Science and Engineering, East West University, Dhaka, Bangladesh \\ Corresponding Authors: Mir Moynuddin Ahmed Shibly, Shamim Ripon \\ Email address: 2016-3-60-057@std.ewubd.edu, dshr@ewubd.edu
}

In this era of advancements in deep learning, an autonomous system that recognizes handwritten characters and texts can be eventually integrated with the software to provide better user experiences. Like other languages, Bangla handwritten text extraction also has various applications such as post-office automation, signboard recognition, and many more. A large-scale and efficient isolated Bangla handwritten character classifier can be the first building block to create such a system. This study aims to classify the handwritten Bangla characters. The proposed methods of this study are divided into three phases. In the first phase, seven convolutional neural networks i.e., CNN-based architectures are created. After that, the best performing CNN model is identified, and it is used as a feature extractor. Classifiers are then obtained by using shallow machine learning algorithms. In the last phase, five ensemble methods have been used to achieve better performance in the classification task. To systematically assess the outcomes of this study, a comparative analysis of the performances has also been carried out. Among all the methods, the stacked generalization ensemble method has achieved better performance than the other implemented methods. It has obtained accuracy, precision, and recall of $98.68 \%, 98.69 \%$, and $98.68 \%$ respectively on the Ekush dataset. Moreover, the use of CNN architectures and ensemble methods in large-scale Bangla handwritten character recognition has also been justified by obtaining consistent results on the BanglaLekha-Isolated dataset. Such efficient systems can move the handwritten recognition to the next level so that the handwritings can easily be automated. 


\section{Convolutional neural network-based ensemble}

\section{2 methods to recognize Bangla handwritten character}

3

4

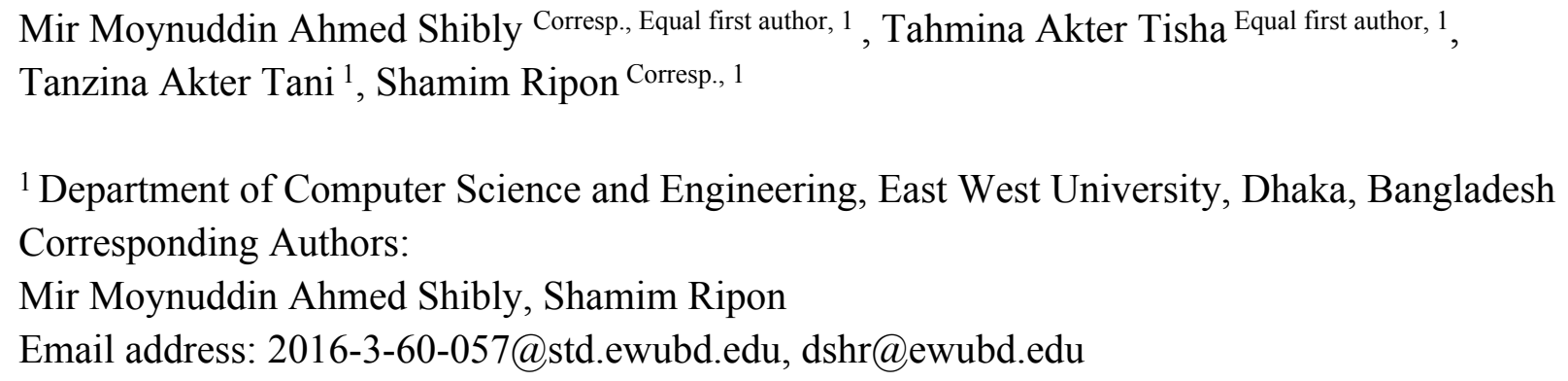

\section{Abstract}

In this era of advancements in deep learning, an autonomous system that recognizes handwritten characters and texts can be eventually integrated with the software to provide better user experiences. Like other languages, Bangla handwritten text extraction also has various applications such as post-office automation, signboard recognition, and many more. A largescale and efficient isolated Bangla handwritten character classifier can be the first building block to create such a system. This study aims to classify the handwritten Bangla characters. The proposed methods of this study are divided into three phases. In the first phase, seven convolutional neural networks i.e., CNN-based architectures are created. After that, the best performing CNN model is identified, and it is used as a feature extractor. Classifiers are then obtained by using shallow machine learning algorithms. In the last phase, five ensemble methods have been used to achieve better performance in the classification task. To systematically assess the outcomes of this study, a comparative analysis of the performances has also been carried out. Among all the methods, the stacked generalization ensemble method has achieved better performance than the other implemented methods. It has obtained accuracy, precision, and recall of $98.68 \%, 98.69 \%$, and $98.68 \%$ respectively on the Ekush dataset. Moreover, the use of CNN architectures and ensemble methods in large-scale Bangla handwritten character recognition has also been justified by obtaining consistent results on the BanglaLekha-Isolated dataset. Such efficient systems can move the handwritten recognition to the next level so that the handwritings can easily be automated.

\section{Introduction}

Bangla is one of the prestigious languages all over the world. About 230 million people from around the world speak Bangla as their native language (Khan, Al Helal \& Ahmed, 2014), and approximately 37 million people use it as a second language for both speaking and writing purposes. Thus, by the total number of speakers worldwide, Bangla is the fifth most spoken native language (Alom et al., 2018) and the seventh most spoken language as well. As Bangla is 
39

40

41

42

43

44

45

46

47

48

49

50

51

52

53

54

55

56

57

58

59

60

61

62

63

64

65

66

67

68

69

70

71

72

73

74

75

76

77

such a renowned language, works related to Bangla language such as Bangla handwritten character recognition is getting more attention among machine learning practitioners. In this age of artificial intelligence and automation, the prominent applications of Bangla handwritten recognition cannot be overlooked. It can play a significant role in many aspects such as post-office automation, national ID number recognition, parking lot management system, and online banking (Alom et al., 2018). This recognition system can also play an essential part in signboard translation, digital character conversation, keyword spotting, scene image analysis, text-to-speech conversion (Manoharan, 2019), meaning translation, and most notably in Bangla optical character recognition (OCR) (Manisha, Sreenivasa \& K., 2016). But it has been a great challenge to provide such a system for Bangla than most other languages. Bangla has a very complex and rich handwriting pattern as opposed to the simple handwriting pattern of other renowned languages. In Fig. 1, a complexity comparison of a Bangla handwritten character has been illustrated i.e., Fig. 1(b), a Bangla handwritten character, has been compared with an English handwritten character i.e., Fig. 1(a), and also with an Arabic handwritten character i.e., Fig. 1(c). From the figure, it is apparent that Bangla characters have a more complex structure than Arabic and English characters.

Bangla script consists of 11 vowels and 39 consonants. These 50 characters are the basic alphabets of the Bangla language. In addition to that, there are more than 170 characters (Das et al., 2014) conjunct-consonant characters that are formed by combining two or more than two basic characters, and these compound characters very close resemblance with each other. For having very complex shaped cursive characters, morphological complexity, the variety of writing styles, and scarcity of the complete Bangla handwritten dataset, recognizing Bangla handwritten characters has become more challenging for a system. To deal with these emerging challenges, researchers have utilized many different methods such as deep convolutional neural networks (DCNNs) (Alom et al., 2018), convolutional neural network (CNN) with transfer learning (Reza, Amin \& Hashem, 2020), and ensemble learning (Rahaman Mamun, Al Nazi \& Salah Uddin Yusuf, 2018).

For the complex patterns that lie into the handwritten Bangla characters, their recognition is a very difficult task. There are various techniques that the researchers have followed in Bangla handwriting recognition challenges. However, convolutional neural network (CNN)-based architectures have proved to be the de-facto standard in this domain of handwritten character recognition (Rahman et al., 2015; Azad Rabby et al., 2018; Chatterjee et al., 2020), and many more. In this study, different convolutional neural networks have been utilized for Bangla handwritten character recognition. On the other hand, ensemble learning is a special method where a model is built with the help of different base classifiers to improve the quality of predictions and overall performance. The ensemble learning methods have been utilized widely for image recognition and classification for a while to achieve better performance. Many studies have explored the possibilities of ensemble methods in image analysis (Ju, Bibaut \& van der Laan, 2018; Das et al., 2018). In light of other works for obtaining outstanding results, in this 
78

79

80

81

82

83

84

85

86

87

88

89

90

91

92

93

94

95

96

97

98

99

100

101

102

103

104

105

106

107

108

109

110

111

112

113

114

115

116

study, various ensemble methods have also been used for classifying Bangla handwritten characters.

This study has developed good performing machine learning models to classify Bangla handwritten characters efficiently. The key research contributions of this study are:

1. This study has developed seven convolutional neural network (CNN)-based models to classify isolated Bangla handwritten characters efficiently.

2. This is one of the first reported works that utilize various ensemble methods to recognize Bangla handwritten characters.

3. This study has classified more Bangla handwritten characters than other related state-of-theart existing works with better performances.

In the following section of this article, an extensive literature review of the related works is presented. After that, the methods, materials, and experimental framework of this study are subsequently presented. Then, the results are described with appropriate discussion, and finally, concluding remarks are demonstrated.

\section{Related Works}

(Alom et al., 2018) have introduced the popular deep convolutional neural networks to recognize Bangla handwritten characters. They have performed their experiments on the CMATERdb (Sarkar et al., 2012). The used architectures have included DenseNet (Huang et al., 2017), AllConv Net (Springenberg et al., 2015), VGG Net (Simonyan \& Zisserman, 2015), FractalNet (Larsson, Maire \& Shakhnarovich, 2019), ResNet (He et al., 2016), and NiN (Lin, Chen \& Yan, 2014). In another work (Chatterjee et al., 2020), to resolve the issue of a higher number of iterations, the authors have used transfer learning with the ResNet50 network in a need of proper training of the model. To make the training faster, a one cycle policy and the varying image sizes methods have been applied as well. An accuracy of $94.3 \%$ at the character level has been achieved by (Bhattacharya et al., 2016). In (Azad Rabby et al., 2018), two datasets have been used for experiments using a CNN-based architecture. An approach of Xception-based ensemble learning has been adopted by (Rahaman Mamun, Al Nazi \& Salah Uddin Yusuf, 2018) to recognize handwritten Bangla digits.

(Rahman et al., 2015) have developed a CNN-based model to classify the basic handwritten characters. (Alif, Ahmed \& Hasan, 2018) have modified ResNet18 architecture by adding a dropout layer for handwritten classification. Further, in (Chowdhury et al., 2019), CNN-based work has been proposed by the authors. (Shopon, Mohammed \& Abedin, 2017) have proposed a CNN-based model to classify only Bangla handwritten digits. Also, a study of handwritten digit classification has been conducted by (Sharif et al., 2017). Apart from CNNs, Bangla handwritten characters have been classified using a harmony search algorithm too (Sarkhel, Saha \& Das, 2015). The modified quadratic discriminant function (MQDF) method has also been used for the recognition task (Pal, Wakabayashi \& Kimura, 2007). A deep network has been used in this domain-specific classification as well (Sazal et al., 2013). This technique varies from the 
117 conventional methods of character preprocessing for the creation of handcrafted characteristics 118 such as loops and strokes.

119 Various research for the recognition of handwritten characters and texts from other languages 120 have also been conducted such as recognizing Baybayin scripts using SVM (Sitaula, Basnet \& 121 Aryal, 2021), analyzing handwritten Hebrew document (Biller et al., 2016), recognizing English 122 handwritings (Pham et al., 2020), and many more. For English handwritten digit and character

123

124

125

126

127

128

129

130

131

132

133

134

135

136

137

138

139

140

141

142

143

144

145

146

147

148

149

150

151

152

153

154

155

156

recognition tasks CNN-based architectures have yielded better performance than other techniques (Baldominos, Saez \& Isasi, 2019; Ranzato et al., 2007; Cireşan et al., 2011), and so on have applied different variants of CNN on the MNIST dataset and obtained good results. Similarly, good results have been obtained on the EMNIST dataset using convolutional neural networks (Peng \& Yin, 2017; Mor et al., 2019).

So far, the described related works for various handwritten character recognition are mostly CNN-based. However, one of the objectives of this study is to explore the usability of ensemble methods for the commenced recognition task. Although the ensemble techniques have not been used vastly for handwritten classification like CNN, they have been used widely in other image classification tasks. (Shibly, Tisha \& Ripon, 2021) has used the stacked generalization method for handwritten character recognition. Before this, SVM based stacked generalization has also been used for image classification (Tsai, 2005). The stacked ensemble generalization approach has already demonstrated the promising performance of disease detection in the medical field (Rajaraman et al., 2018). Stacked generalization has also been used for multiclass motor imagery-based brain-computer interfaces (Nicolas-Alonso et al., 2015). The other ensemble methods like bagging (Hothorn \& Lausen, 2003), boosting (Fidalgo et al., 2018), random forest (Gislason, Benediktsson \& Sveinsson, 2006) have also been implemented in image classification. These ensemble methods have been utilized to improve the performances of the classifiers. The extensive literature review suggests that the convolutional neural network-based architectures are widely used in the recognition of Bangla handwritten characters. But the number of characters recognized by most of the works is limited in comparison with a large number of characters in the Bangla language. This study is an attempt to recognize as many handwritten characters as possible with widely used CNN architectures. Moreover, the literature review also suggests that there is a gap of knowledge about the applicability of ensemble methods in handwritten Bangla character recognition tasks. This study also explores few ensemble methods to improve the recognition performance.

\section{Methods}

In this section of the article, the methodologies of this study are presented with appropriate explanations. The three phases of the applied methodology are described in this section. A highlevel overview of the workflow of this study is illustrated in Fig. 2.

\subsection{Convnet Architectures}

Six popular convnet architecture has been used to classify Bangla handwritten characters. These architectures are AlexNet (Krizhevsky, Sutskever \& Hinton, 2017), VGG16 (Simonyan \&

Peer] Comput. Sci. reviewing PDF | (CS-2021:01:57252:1:1:NEW 27 Apr 2021) 
157

158

159

160

161

162

163

164

165

166

167

168

169

170

171

172

173

174

175

176

177

178

179

180

181

182

183

184

185

186

187

188

189

190

191

192

193

194

195

196

Zisserman, 2015), VGG19 (Simonyan \& Zisserman, 2015), ResNets (He et al., 2016), Xception (He et al., 2016), and DensNet (Huang et al., 2017). Additionally, a VGG-like small convnet has also been developed by us. Each architecture has its unique style, and they try to solve the image classification problem in their own different ways.

The convnets are very useful for extracting features from images. Handcrafted features for image classification are not efficient enough to build robust classifiers. With the help of convolution, which is an attempt to mimic how the brain convolves, a convnet model identifies various shapes that lie in an image. As the final step of convolution, a feature vector of fixed length for every image is generated. After that, those features are used to classify the images. The convnets can also have classification layers on top of the feature extraction layers. For having both feature extraction and classification ability integrated into a single architecture, a convnet is convenient to use. Convolutional neural network-based models have also been proved to be better performers in image classification and recognition. For these reasons, different convnets have been applied for the recognition task. Most importantly, a wide range of convnets has been used to find the optimal model for Bangla handwritten character recognition task.

\subsubsection{AlexNet, VGG16, VGG19, Small CNN}

AlexNet, VGG16, VGG19, and our developed small CNN are four convolutional neural network models that have similar sequential structures. These four convolutional neural networks have been used for recognition tasks for their simple yet very powerful architectures. Among them, AlexNet is one of the very first convnet architectures that utilize graphical performance units (GPU) for processing images. The comparative description of four architectures is presented in Table 1. AlexNet consists of eight layers. The architecture starts with a convolutional layer followed by a max-pooling layer. This convolutional - max-pooling layer combination repeats two times. After that, there are three convolutional layers and a single max-pooling layer. The last three layers of AlexNet are dense while the last one being the output layer. VGG16, VGG19, and small CNN have an AlexNet-like structure. However, the VGG-networks are deeper than AlexNet. VGG16 and VGG19 are sixteen- and nineteen-layers architectures, respectively. On the other hand, the small $\mathrm{CNN}$ architecture has eight layers.

\subsubsection{ResNets}

Residual Networks i.e., ResNets (He et al., 2016) have also been used for the classification challenges. The convolutional and the identity blocks are two building blocks of a ResNet that have been presented in Fig. 3. The ResNets are unique for their residual or skip connections. The information propagates from a specific layer to another specific layer, they follow two paths. In a convolution block, the information goes through a series of convolutional - batch normalization layers. The information goes through a point-wise convolutional layer as well. Then the weights learned from the two paths are added after the end of the convolutional block. Similar things happen in identity block. To solve a sophisticated classification task, a deeper network is needed. But deeper networks tend to fail in learning due to degradation problems. Even sometimes the performance of a network falls due to adding new layers. Various skip connections in ResNets have been employed to prevent this degradation within a network. 
197

198

199

200

201

202

203

204

205

206

207

208

209

210

211

212

213

214

215

216

217

218

219

220

221

222

223

224

225

226

227

228

229

230

231

232

233

234

235

236

A ResNet can be of $18,34,50,101$, or 152 layers. All the ResNets start with a $7 \times 7$ convolutional layer with 64 filters and strides of 2 followed by a batch normalization layer and a $3 \times 3$ maxpooling layer having strides of 2 . After that, for ResNet50 architecture, there is a convolutional block is followed by two identity blocks. Then there is another convolutional block followed by three identity blocks. This pattern continues two more times except with five identity blocks and two identity blocks, respectively. After the last identity block, the outputs are reduced with average pooling, and finally, a SoftMax classification layer is added.

\subsubsection{Xception}

There are skip connections in the Xception architectures as well. Inception network (Szegedy et al., 2015) inspired the creation of this architecture. The modification of this architecture over the Inception is that each inception module has been replaced with a depthwise separable convolutional layer. Just like a typical convolutional layer, the depthwise convolutional layer has the same output. But to reach the same output, a separable convolutional layer requires fewer computations than the regular convolutional layer. Such improvement over the convolutional layer makes a model be trained faster.

In Fig. 4, the Xception architecture is presented. Xception begins with two typical convolutional layers having 32 and 64 filters, respectively with a fixed filter size of $3 \times 3$. This is followed by five Xception blocks. Each input of such a block can either be passed through two separable convolutional layers and a max-pooling layer or can be passed through a pointwise convolution via shortcut connection except the fourth Xception block. The fourth Xception block consists of only one separable convolutional layer, and this block repeats eight times. After the last Xception block, there are two separable convolutional layers followed by a global average pooling layer. After that, the fully connected layers are added with the last being the output layer.

\subsubsection{DenseNet}

Another used convnet in this work is DenseNet. The idea behind this model is that every convolutional layer in a certain block is connected to every other convolutional layer of that block. Every layer not only processes the input forwarded by the immediately preceding layer but also processes the input combination forwarded by all the previous layers. Basically, a DenseNet model consists of a few dense blocks. Before every dense block, there is a convolutional layer followed by an average-pooling layer except the first dense block. The block diagram of DenseNet is displayed in Fig. 5. There is a convolutional layer with a filter size of $7 \times 7$ followed by a max-pooling layer with a pool size of $3 \times 3$ before the first dense block. The other transitional convolutional layers have a filter size of $1 \times 1$. After the last dense block, there are a global average-pooling layer and few customizable classification layers.

\subsection{Image Data Augmentation}

It has been proved that image data augmentation helps a classifier to recognize images more accurately (Perez \& Wang, 2017). Here, while creating the individual CNN models, different types of data augmentation have been applied to the training images. The images have been rotated from 9 degrees to 15 degrees. The height and width shift range also has been adjusted 
237 from 0.09 to 0.15 , and the zoom range is varied from 0.09 to 0.15 . These types of augmentation

238

239

240

241

242

243

244

245

246

247

248

249

250

251

252

253

254

255

256

257

258

259

260

261

262

263

264

265

266

267

268

269

270

271

272

273

274

275

276

provide more generalization to the classifiers. Different image augmentations used in this study are presented in Table 2 considering $A_{1}$ as rotation range, $A_{2}$ height shift range, $A_{3}$ as width shift range, and $A_{4}$ as zoom range.

\subsection{Convnet as Feature Extractor}

The described convnets can be used as feature extractors as well. The feature extractor model can be developed by removing the fully connected layers from a convnet. The flatten layer after the last pooling layer produces feature vectors. These can be used to train a separate classifier. Among the described architectures, ResNet50 has shown the highest accuracy in the classification task. This convnet has been used as a feature extractor in this work to train separate classifiers with traditional machine learning algorithms, such as Logistic Regression, Decision Tree, Naïve Bayes, and Support Vector Machine. Using a pre-trained ResNet50 feature extractor, features from train and test images have been extracted. The flatten layer of the model produces a feature vector of size 2048. Fitting a classifier on this huge feature space is not feasible due to a lack of computational resources. To overcome this issue, three dense layers with 1024, 512, and 80 neurons have been added before the final classification layer. This has been done to narrow down the feature space to a manageable size.

\subsection{Ensemble Methods}

The classification task has also been carried out using some ensemble techniques. In ensemble learning, a prediction has been made based on multiple learning algorithms rather than single learning algorithms (Opitz \& Maclin, 1999). It is a technique used in machine learning fields where more than one model is trained for the same task as opposed to a typical machine learning technique for solving a particular task. In ensemble learning, hypotheses from different models are combined to create a more generalized, accurate, and robust model to solve a specific problem. Few ensemble techniques can develop a generalized model from multiple models. Regarding this, the most commonly used ensemble techniques are majority voting, weighted majority voting, Borda count, bagging, boosting, stacked generalization, and so on. One of the objectives of this study is to explore the potential of ensemble methods in Bangla handwritten character recognition. Regarding this, in this study, stacked generalization, bootstrap aggregating, adaptive boosting, extreme gradient boosting, and random forest ensemble methods have been used for the handwritten recognition task.

\subsubsection{Stacked Generalization}

Stacked generalization follows a very general process. The mechanism of this method is illustrated in Fig. 6. In this method, on the training dataset, $\mathrm{k}$ different first-level classifiers are fitted. There are many methods to obtain these first-level classifiers (Aggarwal, 2015). In this work, a 10-fold cross-validation method has been employed on a training set, and ten first-level classifiers have been developed. The data augmentation technique for each first-level classifier 
277 has been presented in the figure. After obtaining first-level classifiers, they are stacked, and their 278 outputs are concatenated. After the concatenation, three dense layers have been added. The last 279 layer of the second-level stacked model is the output layer. After creating the stacked model, it 280 has been trained with the validation set, where the output of each first level classifier works as 281 the new dataset for training the second level classifier. This is a lesser-used method for image 282 classification with great potential. To explore its usability and to improve recognition 283 performance, this method has been employed.

284

285

286

287

288

289

290

291

292

293

294

295

296

297

298

299

300

301

302

303

304

305

306

307

308

309

310

311

312

313

314

315

316

\subsubsection{Bootstrap Aggregating}

The second ensemble method that has been employed is bootstrap aggregating. It is also known as bagging. In bagging, from training data, a portion of images are selected randomly and put in a bag with replacement. Usually, $1-1 / \mathrm{e}=63.2 \%$ instances from the training set go to a bucket with the other $36.8 \%$ of the copied instances from randomly selected data. By doing this, a bag with the size of the original training set is constructed. This is the principal idea behind bootstrap aggregating. In Fig. 7, the overall working procedure of this method has been demonstrated along with the data augmentation technique applied on each classifier. After creating ten bags from the training dataset, an independent classifier has been fitted on each bag. And for testing, majority voting has been applied to the predictions made by individual classifiers. This method is an attempt to reduce variance from the classifier (Aggarwal, 2015). Like stacked generalization, this method has also been used to achieve better performance.

\subsubsection{Boosting and Random Forest}

Two boosting methods have been used in this work - adaptive boosting and extreme gradient boosting. In AdaBoost, the classification starts with equal weight to each of the training instances. The weight associated with an instance indicates the probability of being chosen in bootstrap for training on a certain iteration. If an instance is misclassified, the weight associated with that instance increases for the next iteration. If an instance is correctly classified, the weight gets decreased. The iteration of training repeatedly terminates when the classifier's accuracy becomes $100 \%$ or the classifier performs worse than the base classifier (Aggarwal, 2015). Another boosting method is XGBoost (Chen \& Guestrin, 2016). It is a tree-based ensemble technique where the errors are minimized by taking extreme measures using a gradient descent algorithm.

The last ensemble technique that has been used is the random forest. A random forest is nothing but a combination of many decision trees. In this method, $\mathrm{k}$ decision trees are created using training data to form a forest, and for the test data, the majority voting technique is followed. The majority class predicted by individual decision trees is the predicted class. The trees are not developed with all features. Another way to create a random forest to use a bootstrap aggregating technique (Han, Kamber \& Pei, 2012). A portion of the training data points are selected with replacement, and for each decision tree, a newly sampled training dataset is used. Random forest resolves the problem of overfitting in decision tree classifiers (Vinet \& Zhedanov, 2010). 


\section{4. Experimental Setup}

318 This study aims at classifying Bangla handwritten characters. For creating the convnets, Keras 319 ("Keras") on top of TensorFlow ("TensorFlow") - a python library has been used. For a few of 320 the experimentations, the sci-kit-learn ("scikit-learn") library of python has also been utilized.

321 The models have been trained on a computer having Ryzen 5, 1600CPU, 8GB ram, and Nvidia

322 GeForce 1050TI GPU with Linux Mint 19.3 operating system.

323

324

325

326

327

328

329

330

331

332

333

334

335

336

337

338

339

340

\subsection{Dataset}

We have used two publicly available Bangla handwritten character datasets for the experiments Ekush (Rabby et al., 2019) and BanglaLekha Isolated (Biswas et al., 2017). Primarily, the experiments have been conducted on the Ekush dataset. For comparative analysis purposes, the experiments have been carried out on the BanglaLekha-Isolated dataset too. There are other datasets too like ISI (Chaudhuri, 2006), NumtaDB (Alam et al., 2018), and CMATERdb (Sarkar et al., 2012). But they do not have as many classes as Ekush and BanglaLekha-Isolated datasets. Ekush dataset has the highest number of classes among all Bangla handwritten character datasets. It has 122 classes of characters of four types like modifiers, basic characters, compound characters, and numerals. This dataset contains 734,036 different images. The creators of this dataset have collected handwritten images in a form from 3,086 people among which $50 \%$ are male and $50 \%$ are female. Table 3 shows the details of the Ekush dataset. On the other hand, the BanglaLekha-Isolated dataset has 84 classes distributed in 50 basic characters, 10 numerals, and 24 compound characters. The images of both datasets are greyscaled images. The images of the Ekush dataset have a fixed dimension of $28 \times 28$ pixels whereas the dimension of the images of the BanglaLekha-Isolated dataset varies from $36 \times 36$ pixels to $191 \times 191$ pixels. During experiments, images from both datasets have been kept with a fixed dimension of $28 \times 28$.

341 In Fig. 8(a), few vowel modifiers from the Ekush dataset are displayed. The vowel modifiers are labeled from 0 to 9 in the dataset. These characters cannot be found isolated. They are found before or after, or both before and after consonants or compound characters. These are the representative or short forms of actual vowels. There are eleven vowels in the Bangla language. They are labeled from 10 to 20 . These characters can be in a word independently. But if they are to be used as phonetics, their corresponding vowel modifiers work on the consonants and compound characters. Few of the vowels are displayed in Fig. 8(b). In Fig. 8(c), a few of the 39 consonants of the Bangla language are displayed. They are labeled from 21 to 59 . A few of the 52 compound characters of the Ekush dataset are shown in Fig. 8(d). They are labeled from 60 to 111. Compound characters in Bangla can be constructed by joining two or more consonants. Finally, in Fig. 8(e), four of the ten Bangla handwritten digits of this dataset are shown. They are labeled from 112 to 121.

\subsection{Training, Testing, and Validating}

355

The datasets have been divided into three sets - train, validation, and test. The train set has 356 approximately $75 \%$, the validation set has approximately $10 \%$, and the test set has approximately $15 \%$ of the data. For the Ekush dataset, 547,131 of 729,750 images in the whole dataset are used 
357 for training. And for validation and test, the number of images is 72,842 and 109,777 ,

358

359

360

361

362

363

364

365

366

367

368

369

370

371

372

373

374

375

376

377

378

379

380

381

382

383

384

385

386

387

388

389

390

391

392

393

394

395

396

respectively. For the BanglaLekha-Isolated dataset, for training convnets, 224, 211 images have been used. And in the validation and test sets, there have been 24,881 and 33,310 images, respectively. For training the first level classifiers of the stacked generalization model, the training set has been divided into ten folds using 10 -fold cross-validation and a classifier on each fold has been fitted. After combining the first-level classifiers, the second-level classifier has been trained with the validation set of the original dataset.

The reason behind not using the training set again is that the first-level classifiers have already known the training set. That is why the original validation set which is completely unknown for the first level classifiers has been used. After training the second level classifier, it has been tested against the original test images - the same test images that have been used for testing throughout the study. For the bootstrap aggregating method, train and validation sets have been combined. From the combined set, ten bags of datasets have been sampled with replacement using the bootstrap aggregating method. After fitting a convnet on each of the bags, the final classifier (based on the results of ten convnets) has been tested with the original test set.

\subsection{Optimizers, Loss Function, Batch Size, and Evaluation Metrics}

To select appropriate optimizers, few models have been trained with various optimizers. The optimizer that has the best performance has been used for model training. Similarly, preexperiments with various batch sizes have also been conducted and the best performing batch size has been used for actual model creation. As the models have to accomplish a multi-class classification task, the categorical cross-entropy loss function has been used. Moreover, four evaluation metrics have been used throughout the whole working process of this studyprecision, recall, F1-score, and accuracy.

\section{Results}

The overall performances of the experiments are generally well and competitive to other existing works. In the previous section, the three stages of the experimental setup have been described. In the first stage, the different convnets have demonstrated results ranging from $96.25 \%$ accuracy to 97.81\% accuracy on the Ekush dataset. For the BanglaLekha-Isolated dataset, accuracies have varied from $88.88 \%$ to $93.55 \%$. The most accurate performance that has been recorded is $98.68 \%$ in terms of accuracy for the Ekush dataset. This has been obtained by the stacked generalization ensemble method. For the BanglaLekha-Isolated dataset, the highest achieving performer has been the bootstrap aggregating method with $93.55 \%$ test accuracy. Apart from the classifiers' performances, other aspects are needed to be discussed too. In this section, the results of the study along with various aspects are presented elaborately with appropriate discussion.

\subsection{Result Comparison}

The result of this study has outperformed most of the existing works in the domain of Bangla handwritten character classification. In Table 4, a comparison of this work with other existing works has been presented after an extensive literature review. The comparison has proven that

Peer] Comput. Sci. reviewing PDF | (CS-2021:01:57252:1:1:NEW 27 Apr 2021) 
397

398

399

400

401

402

403

404

405

406

407

408

409

410

411

412

413

414

415

416

417

418

419

420

421

422

423

424

425

426

427

428

429

430

431

432

433

434

435

436

our work has exemplary and outstanding results in terms of better performances and classifying Bangla handwritten characters on a large scale as well. Most of the current works have classified less than 122 characters. Very few of them have classified 122 or more than 122 Bangla

handwritten characters. In contrast, this study has beaten (Azad Rabby et al., 2018) which has the same dataset as ours. They have the same train-test split as our experiments with $15 \%$ images in the test set. They have reported a $97.73 \%$ test accuracy obtained by their EkushNet architecture whereas our stacked generalization method has been able to achieve $98.68 \%$ test accuracy. Our developed bootstrap aggregating method has also outperformed the performance of the EkushNet model.

(Bhattacharya et al., 2016) and (Pal, Wakabayashi \& Kimura, 2007) have classified 152 and 138 handwritten characters, respectively but have not gained performance like our results. We have achieved a maximum of $98.68 \%$ accuracy for the classification task while the other works' performances have not exceeded $98 \%$ accuracy. To further validate our obtained results, the proposed methods have also been applied on the BanglaLekha Isolated dataset (Biswas et al., 2017) which has the second-highest number of Bangla isolated characters among all publicly available datasets in this domain. Our proposed models on this dataset have also outperformed other works. Stacked generalization and bootstrap aggregating methods have yielded $92.67 \%$ and 93.55\% test accuracy whereas (Purkaystha, Datta \& Islam, 2017) have obtained $88.93 \%$ test accuracy. They also have not considered all 84 classes of the dataset rather they have built their model on 80 classes which further proves the superiority of our methods as all 84 classes have been used for classification in our work.

\subsection{Performances of Convnets}

During training, after each epoch, the training and validation accuracies of the convnets with their respective losses have been observed to check on the stability of models. The models have been trained for a hundred epochs, and at the end of each epoch, they have been validated against the validation images. In Fig. 9, the validation accuracies over the number of epochs for each convnets for the Ekush dataset have been compared. The figure depicts that all seven models have generally smooth learning curves. The comparison of the validation accuracies of the models indicates that the ResNet50 model has been better than other models. And, the DenseNet model has been worse than others. The Xception model has a performance almost like the ResNet50, and AlexNet has a performance close to DenseNet. The other three models - small CNN, VGG16, and VGG19 are somewhere in between good and bad.

On the other hand, Fig. 10 depicts the validation loss over the epochs of each convnet for the Ekush dataset. Unlike validation accuracy curves, the validation loss curves of convnets are not smooth. The validation loss of the small CNN model is lesser than other models in a certain epoch while DenseNet has a higher loss than others. The small CNN and DenseNet models have minimum losses of 0.0459 and 0.1841 , respectively. These experimental values indicate that the small CNN model has been more stable than other models in terms of validation loss, and the DenseNet model has been more unstable than others. The other models have stability between

Peer] Comput. Sci. reviewing PDF | (CS-2021:01:57252:1:1:NEW 27 Apr 2021) 
437 the two discussed models. Moreover, the ResNet50 model has outperformed all the other six 438 models with $97.81 \%$ test accuracy. It has also $97.82 \%, 97.81 \%$, and $97.81 \%$ precision, recall, 439 and F1-score, respectively.

440 All the convnets' precision, recall, F1-score, and accuracy on the test set of the Ekush dataset are

441

442

443

444

445

446

447

448

449

450

451

452

453

454

455

456

457

458

459

460

461

462

463

464

465

466

467

468

469

470

471

472

473

474

475

476

given in Table 5. From there, it can be observed that the Xception model has come close to ResNet 50 with $97.63 \%$ accuracy, $97.64 \%$ precision, and $97.63 \%$ recall. While these two models have performed better, VGG16, VGG19, and small CNN models have average performances with accuracies of $96.97 \%, 97.05 \%$, and $97.30 \%$, respectively. On the contrary, DenseNet and AlexNet have comparatively poor performances than others with accuracies of $96.25 \%$, and $96.80 \%$, respectively. The reason behind the good performance of ResNet50 and Xception is that they have very deep and complex structures. These architectures have been able to extract the patterns of Bangla handwritten characters efficiently. It is also to be noted that the poorperforming models such as DenseNet and AlexNet have also taken more time to train than the models with better performance. They have also been slower than the better-performing models like ResNet50 and Xception in the testing phase. The performances of convnets on the BanglaLekha-Isolated dataset are also presented in Table 5. Among the convnets, the ResNet50 model has been the top performer with $92.63 \%$ test accuracy while the AlexNet model has yielded only $88.88 \%$ accuracy.

\subsection{Performances of ResNet as Feature Extractor}

In the second phase of the study, the best performing convnet has been used as a feature extractor for the classification task. The goal of this experiment has been to explore the applicability of regular classification algorithms in image classification. As it is known that the images are subject to contextual information. Therefore, simply passing the original pixel values of images into a classifier without giving additional information would not yield better performance. To use those classifiers, contextual features from images are needed to be extracted. For feature extraction purposes, the best pre-trained best-performing convnet has been used by removing its classification layers - in this case, that is ResNet50. As a convnet has been used, the performance of the classifiers depends on how well the convnet can extract features from images. Better convnet will help the classifiers to obtain better performance.

In Table 5, for both datasets, the performances of logistic regression, support vector machine, naïve Bayes, and decision tree classifiers with pre-trained ResNet50 as feature extractor has been presented. Among them, the SVM classifier performed better than other classifiers with $97.75 \%$ accuracy, $97.76 \%$ precision, and $97.75 \%$ recall on the Ekush dataset. On the other hand, logistic regression has been the top performer on the extracted features with $92.17 \%$ accuracy, $92.24 \%$ precision, and $92.17 \%$ recall. Although the shallow classifiers could not beat the original ResNet50 classifier which has $97.81 \%$ accuracy, they have worked exceptionally well with a very small feature set for the Ekush dataset. The same minor downgrade in performances of shallow classifiers from the original ResNet50 model has been observed for the BanglaLekhaIsolated dataset.

Peer] Comput. Sci. reviewing PDF | (CS-2021:01:57252:1:1:NEW 27 Apr 2021) 
477 5.4. Results of Ensemble Methods

478 In the final stage of the experiments, few ensemble methods have been employed for the 479 classification task. The ensemble methods are broadly categorized into two types. One type of 480 ensemble method is where the classifiers have been built from scratch on the original training 481 images, and the other type is where the classifiers have been trained on the training set with 482 extracted features by the ResNet50 model. The classifiers of the first type of ensemble method 483 have comparatively better performances than the second. Even they have demonstrated better 484 performances than all the other methods employed during this study.

485

486

487

488

489

490

491

492

493

494

495

496

497

498

499

500

501

502

503

504

505

506

507

508

509

510

\subsubsection{Performance of Stacked Generalization Ensemble Method}

To create the second-level ensemble classifier, ten different classifiers have been trained initially. After training the first-level classifiers, they have been tested with the original test images. In Fig. 11, their performances on the Ekush dataset are presented. The performances of first-level classifiers have a resemblance with their original convnets. ResNet50 and Xception models have better performances than others, and AlexNet has the lowest performance. The best first-level classifier has been the ResNet50 model that has been trained on the $7^{\text {th }}$ fold with rotation range $=$ 14 , height shift range $=0.10$, width shift range $=0.10$, and zoom range $=0.10$. It has a test accuracy of $97.90 \%$ which is better than its ResNet50 counterpart trained on the whole training set. This better-performing trend only has been observed in the ResNet models.

The other models have lost some performance for being trained on a subset of the original training set. The stacked generalization model has outperformed the other individual classifiers with $98.68 \%$ test accuracy. Using different models, different image augmentations, and a different subset of the training set on each first level classifier has helped to achieve better performance of the second level classifier. Precision, recall, F1-score, and the accuracy comparison of first and second-level classifiers on the Ekush dataset are given in Fig. 11. From the figure, it can be seen that the stacked model has the highest performance in all the evaluation metrics. The stacked model has $98.69 \%$ precision, $98.68 \%$ recall, $98.68 \% \mathrm{~F} 1$-score, and $98.68 \%$ accuracy on the Ekush dataset while the first level models do not have the same level of performance. On the other hand, from Table 5, the stacked model has achieved $92.78 \%$ precision, $92.67 \%$ recall, $92.72 \% \mathrm{~F} 1$-score, and $92.67 \%$ accuracy on the BanglaLekha-Isolated dataset.

511 ten individual classifiers. Each of them has been trained on one of the ten bags of training

512 images. Figure 12 shows these ten models' test performances on the Ekush dataset. Like the

513 first-level classifiers of the stacked generalization method, the individual classifiers of the 514 bootstrap aggregating method have similar performances. ResNet50 model with image 515 augmentation of rotation range $=10$, height shift range $=0.1$, width shift range $=0.1$, and zoom 516 range $=0.1$ has been the better performer than others. 
517 AlexNet model has worse performance than other models. It can also be seen from the figure that 518 other ResNet18, VGG16, and Xception models have come close to ResNet50 in terms of test 519 accuracy. After finishing the training of these convnets, the test images have been tested using 520 the majority voting method on the output of ten convnets. The performance of the predictions 521 based on ten models has outperformed all the individual models. The evaluation metrics-wise 522 comparison of individual models and bagged models has been shown in Fig. 12 as well. The 523 figure depicts that the bagging method has yielded $98.38 \%, 98.37 \%, 98.37 \%$, and $98.37 \%$ 524 precision, recall, F1-score, and accuracy, respectively. On the other hand, the individual models 525 do not have any value of evaluation metrics larger than $97.5 \%$. This increase in model

526

527

528

529

530

531

532

533

534

535

536

537

538

539

540

541

542

543

544

545

546

547

548

549

550

551

552

553

554

555

556

performances of both stacked generalization and bagging methods justifies the efficiency of these classification methods.

The bootstrap aggregating method has also obtained superior performances than individual convnets for the BanglaLekha-Isolated dataset. From Table 5, it can be said that the bagging method has achieved $93.60 \%$ precision, $93.55 \%$ recall, $93.55 \% \mathrm{~F} 1$-score, and $93.55 \%$ accuracy. In fact, the bagging method has achieved the best result among all the other experiments on the BanglaLekha-Isolated dataset.

\subsubsection{Performance of Boosting and Random Forest}

Among the other ensemble classifiers, the random forest has performed better than the other two for both datasets. The precision, recall, F1-score, and accuracy of these models are demonstrated in Table 5. The random forest has secured first place with $97.32 \%$ accuracy, $97.33 \%$ precision, and $97.32 \%$ recall on Ekush. On the other hand, AdaBoost has worse performance than XGBoost with $96.37 \%$ accuracy, $96.42 \%$ precision, and $96.36 \%$ recall while XGBoost has $96.58 \%$, $96.59 \%$, and $96.58 \%$ accuracy, precision, and recall, respectively on the Ekush dataset.

\subsection{Ablation Studies}

Batch size and optimizers are two important parameters when it comes to large-scale image classification. As the task of image classification is very computationally expensive, choosing the appropriate batch size and optimizer can play significant roles in model performance. For this purpose, a few pre-experiments with batch size and optimizers have been carried out. For choosing a batch size, a small CNN model has been developed and trained for 40 epochs with various batch sizes. From the experiments, it has been observed that a larger batch size usually helps the model to obtain better performance in terms of test accuracy. Experiment with 1024 batch size has yielded better performance than that of 512 batch size on Ekush dataset. The larger batch sizes do not always yield better performance either. Classifiers with 1536 or 2048 batch sizes have performed less accurately than that of 1024 batch sizes.

In the second ablation study, a ResNet50 model has been developed and trained for ten epochs with five optimizers - adam, RMSprop, stochastic gradient descent (SGD), Adagrad, and Adadelta. Among different optimizers, SGD has performed worse than other optimizers in terms of validation accuracy (89.52\%). RMSprop has higher validation accuracy than others (96.48\%).

PeerJ Comput. Sci. reviewing PDF | (CS-2021:01:57252:1:1:NEW 27 Apr 2021) 
557 As the batch size of 1024 and RMSprop optimizer have better performance in the pre-

558 experiments, this combination of batch size and optimizer has been used for the rest of the

559 experiments.

560 Time is another important feature that must have to be considered when it comes to model

561 evaluation. Although training time is often overlooked if the model can classify an instance in a

562 real-world environment faster. But due to a lack of available computational resources, training a

563 convnet can become time-consuming. With the experimental setup of this study, the VGG19

564 model has taken on an average 0.3271 millisecond time to process an image while training. This

565 is the lowest time among the seven convnets. In Table 6, the time to process an image by each

566 model, and time to predict an image are given. It can be seen in the table that the DenseNet

567 model has taken more time than the other models to process an image during training. AlexNet

568 has also taken a comparatively longer time to be trained.

569 On the other hand, the VGG16 model has the lowest average prediction time per image. It takes

5700.1572 milliseconds to classify an input image. Besides, the DenseNet model needs more time

571 than any other model to predict an image $(0.4307 \mathrm{~ms})$. Another interesting thing is that all the

572 models tend to work faster in the testing phase than in the training phase except for VGG19.

573 VGG19 has taken 0.336 milliseconds to test an image while it has taken 0.3271 milliseconds to

574 process an image during training. The most significant improvements have been seen in the time

575 of DenseNet to predict. It predicts in 1 millisecond less than the training time per image. The

576 other models have shown improvements in testing time too. However, in terms of performance-

577 time trade-off, ResNet50 and Xception have been better classifiers. They have achieved better

578 test accuracy than other models with relatively faster predictions. It is also a point to be noted

579 that all the ablation experiments have only been applied on Ekush dataset under the assumption

580 that a similar pattern of performances will be found for the BanglaLekha-Isolated dataset.

581

582 6. Discussion

583 Among the individual convnets, the ResNet50 model has the best performance for the Bangla

584 handwritten recognition task for both datasets. And among the regular classifiers using ResNet50

585 as a feature extractor, the SVM classifier has the best performance for both datasets. And,

586 finally, among the ensemble methods, stacked generalization has the best performance for the

587 Ekush dataset while the bootstrap aggregating model has the best performance on the

588 BanglaLekha-Isolated dataset. Among all the classifiers, the stacked generalization and bootstrap

589 aggregating methods have produced the best classifiers for two datasets, respectively. Two of the

590 seven convnets have performed with the best accuracies on the primary dataset of our

591 experimental setup. Those convnets are the ResNet50 and the Xception network. Similarly, two

592 of the ensemble methods have the highest performances. Stacked generalization ensemble and

593 bootstrap aggregating methods have been proven to be exemplary in the field of Bangla

594 handwritten recognition. These ensemble methods have not been used in this domain-specific

595 classification task before. Additionally, the convnets have been used as feature extractors too.

596 Moreover, various experiments have been carried out to tune the different aspects related to this 
597

598

599

600

601

602

603

604

605

606

607

608

609

610

611

612

613

614

615

616

617

618

619

620

621

622

623

624

625

626

627

628

629

630

631

632

633

634

635

636

study too. These types of classifiers can be used for handwritten automation in various realworld applications.

In the first phase of the study, on the primary dataset, the reason for ResNet50 and Xception models having better results is their complex and sophisticated structures. For having more than 120 classes in the dataset, and for the complexity of various handwritten Bangla characters as well, the classification requires more robust and advanced architectures. As mentioned, these two models have more robust and deeper architectures than most of the other convnets that have been used, they have been able to yield outstanding performances. The training time has also been faster for them as they utilize their shortcut connections. Another aspect is to discuss the excellent performances of stacked generalization ensemble and bootstrap aggregating methods. To build classifiers using these two methods, individual convnets have been trained separately. When the final classifier predicts an image into a specific class, it uses all those bits of knowledge learned from several convnets instead of a single model. Those several convnets are also trained differently one from another with different architectures and with different image augmentation. Using these wide ranges of model learnings has allowed stacking and bagging methods to perform exceptionally better than single convnets.

However, the other ensemble methods have not performed as well as stacking and bagging. For the other ensemble methods, the dataset after extracting features using ResNet50 has been used. The performance generally relies on the feature extraction process. Better feature extraction helps to yield better model performances. As the other ensemble classifiers have been trained on the features extracted by ResNet50, it is obvious that they will not be able to beat the original ResNet50 model. A similar justification is applicable for worse performances by logistic regression, SVM, naïve Bayes, and decision tree classifiers. Nevertheless, achieving a respected result with fewer features (80 instead of 1024) is a positive outcome.

\subsection{Misclassification}

After testing the convnets, their confusion matrices and class-wise classification performances have been observed. In this large-scale classification, the classifiers have performed poorly on a few classes from 122 classes of Bangla handwritten characters from the Ekush dataset. Although the classifiers have good performance overall, they have not been able to perform better for a few classes. All convnets have demonstrated a similar trend in misclassifying instances from a few specific classes. In Table 7, a few examples of classes where the ResNet50 classifier has shown poor, average, and good performance with their supports are presented with the number of false negatives, and the number of false positives.

There are some reasons behind failing to perform better for some specific classes. One of them is the lack of a sufficient number of training and testing images in a class. For example, a handwritten character labeled as 111 has fewer training and testing instances. The lower support value for this class from Table 7 indicates the insufficiency of the number of instances during testing. This class has only 1237 training images, while the other classes have more than 4500 images each on average. Although, this is not only the reason for poor performance. Similar 
637 patterns of Bangla handwritten characters across different classes are also responsible. There are 638 few characters in Bangla that have almost the same pattern. A few of the examples are given in

639 Fig. 13. Character labeled as 111 has similarity with character labeled as 69, class 19 has

640 similarity with class 84 , and so on. These close resemblances between two classes make a 641 classifier predict one class into another. The empirical data also supports that. Among the 47

642 false-negative instances for class 111, 23 of them are misclassified as class 69. And among 32 643 false-positive instances, 15 of them are from class 69. Similar misclassifications have happened 644 for other classes that are presented in Table 7 under the "poor" category. For having similar 645 patterns, often people mistake one character for another. Thus, this results in writing them 646 wrongly. For this reason, there are also some wrongly labeled images in the dataset. For

647

648

649

650

651

652

653

654

655

656

657

658

659

660

661

662

663

664

665

666

667

668

669

670

671

672

673

674

675

676 example, from Fig. 13, the image from the left labeled as 19, and the corresponding image from the right labeled as 84 are the same characters but labeled as different classes. Moreover, for those that are categorized under average and good performance, the ResNet50 classifier does not have a majority portion of misclassified instances that are in a specific class, rather the misclassified instances are evenly distributed for the classifier.

\section{Conclusion and Future Works}

Through various experiments, the applicability of convolutional neural network-based ensemble learning methods for handwritten character recognition has been proved. The empirical evidence shows that predictions are more accurate with the models that combine results of multiple convnets than a single convnet. Single convnets may not have the best performance of this work but the robust architectures among them surely have demonstrated impressive results. The ResNet50 and the Xception models have standout performances in the image recognition tasks. The outstanding results that have been attained are also among the top performances that have been reported in the domain of Bangla handwritten character recognition. Another significant aspect is to classify more than 120 handwritten characters. Recognizing them on such a large scale and with such good performance is the major contribution of this work.

However, there are some limitations associated with this study. Only six of the popular convolutional neural networks have been used. The other convnets like Inception (Szegedy et al., 2015), FractalNet (Larsson, Maire \& Shakhnarovich, 2019), NiN (Lin, Chen \& Yan, 2014), etc. can be used for classification tasks too. Additionally, the training of models from scratch can be a tedious job. Exploring the applicability of the pre-trained transfer learning models in the classification task can be a new research direction. Bangla is a very rich language. There are more than 200 compound characters in it. Among them, only 52 compound characters are classified in this study. There is a lack of an available compound character dataset. A dataset with more compound characters can be curated. In Ekush (Rabby et al., 2019) dataset, there are few wrongly labeled images. Those are needed to be labeled properly. Additionally, only five of the ensemble methods have been used for the classification challenge. The other ensemble methods can be applied for this classification task as well. An important aspect of the ensemble methods is that for boosting and random forest, the reduced dataset has been used. For this 
677 reason, the expected performance has not been achieved. To achieve better performance, the 678 classifiers using these methods are needed to be trained from scratch directly on the training 679 images like stacked generalization and bagging ensemble methods. Doing these can produce 680 better performance. Moreover, the applicability of developed classifiers in this study is only 681 limited to isolated handwritten characters. The systems that can recognize words and sentences 682 from images are needed to be created. Extracting words and sentences from images is also very 683 important. Those systems can help us to obtain true autonomous experience in the field of 684 handwriting recognition and extraction.

685

\section{References}

Aggarwal CC. 2015. Data Mining. Cham: Springer International Publishing. DOI: 10.1007/9783-319-14142-8.

Alam S, Reasat T, Doha RM, Humayun AI. 2018. NumtaDB - Assembled Bengali Handwritten Digits. : $1-4$.

Alif MAR, Ahmed S, Hasan MA. 2018. Isolated Bangla handwritten character recognition with convolutional neural network. 20th International Conference of Computer and Information Technology, ICCIT 2017 2018-Janua:1-6. DOI: 10.1109/ICCITECHN.2017.8281823.

Alom MZ, Sidike P, Hasan M, Taha TM, Asari VK. 2018. Handwritten Bangla Character Recognition Using the State-of-the-Art Deep Convolutional Neural Networks. Computational Intelligence and Neuroscience. DOI: 10.1155/2018/6747098.

Azad Rabby AKMS, Haque S, Abujar S, Hossain SA. 2018. Ekushnet: Using convolutional neural network for Bangla handwritten recognition. Procedia Computer Science 143:603610. DOI: $10.1016 /$ j.procs.2018.10.437.

Baldominos A, Saez Y, Isasi P. 2019. A survey of handwritten character recognition with MNIST and EMNIST. Applied Sciences (Switzerland). DOI: 10.3390/app9153169.

Bhattacharya S, Maitra D Sen, Bhattacharya U, Parui SK. 2016. An end-to-end system for bangla online handwriting recognition. In: Proceedings of International Conference on Frontiers in Handwriting Recognition, ICFHR. DOI: 10.1109/ICFHR.2016.0076.

Biller O, Rabaev I, Kedem K, Dinstein I, El-Sana JJ. 2016. Evolution maps and applications. PeerJ Computer Science 2016:1-20. DOI: 10.7717/peerj-cs.39.

Biswas M, Islam R, Shom GK, Shopon M, Mohammed N, Momen S, Abedin A. 2017. BanglaLekha-Isolated: A multi-purpose comprehensive dataset of Handwritten Bangla Isolated characters. Data in Brief. DOI: 10.1016/j.dib.2017.03.035.

Chatterjee S, Dutta RK, Ganguly D, Chatterjee K, Roy S. 2020. Bengali Handwritten Character Classification Using Transfer Learning on Deep Convolutional Network. In: Lecture Notes in Computer Science (including subseries Lecture Notes in Artificial Intelligence and Lecture Notes in Bioinformatics). DOI: 10.1007/978-3-030-44689-5_13.

Chaudhuri BB. 2006. A complete handwritten numeral database of Bangla-A major Indic script. In: 10th International Workshop on Frontiers of Handwriting Recognition (IWFHR), La Baule, France.

Chen T, Guestrin C. 2016. XGBoost: A scalable tree boosting system. In: Proceedings of the ACM SIGKDD International Conference on Knowledge Discovery and Data Mining. DOI: 10.1145/2939672.2939785.

Chowdhury RR, Hossain MS, U1 Islam R, Andersson K, Hossain S. 2019. Bangla handwritten character recognition using convolutional neural network with data augmentation. 2019 
722

723

724

725

726

727

728

729

730

731

732

733

734

735

736

737

738

739

740

741

742

743

744

745

746

747

748

749

750

751

752

753

754

755

756

757

758

759

760

761

762

763

764

765

766

767

Joint 8th International Conference on Informatics, Electronics and Vision, ICIEV 2019 and 3rd International Conference on Imaging, Vision and Pattern Recognition, icIVPR 2019 with International Conference on Activity and Behavior Computing, ABC 2019:318-323. DOI: 10.1109/ICIEV.2019.8858545.

Cireşan DC, Meier U, Gambardella LM, Schmidhuber J. 2011. Convolutional neural network committees for handwritten character classification. In: Proceedings of the International Conference on Document Analysis and Recognition, ICDAR. DOI: 10.1109/ICDAR.2011.229.

Das N, Acharya K, Sarkar R, Basu S, Kundu M, Nasipuri M. 2014. A benchmark image database of isolated Bangla handwritten compound characters. International Journal on Document Analysis and Recognition. DOI: 10.1007/s10032-014-0222-y.

Das A, Roy S, Bhattacharya U, Parui SK. 2018. Document Image Classification with IntraDomain Transfer Learning and Stacked Generalization of Deep Convolutional Neural Networks. In: Proceedings - International Conference on Pattern Recognition. DOI: 10.1109/ICPR.2018.8545630.

Fidalgo E, Alegre E, González-Castro V, Fernández-Robles L. 2018. Boosting image classification through semantic attention filtering strategies. Pattern Recognition Letters. DOI: $10.1016 /$ j.patrec.2018.06.033.

Gislason PO, Benediktsson JA, Sveinsson JR. 2006. Random forests for land cover classification. In: Pattern Recognition Letters. DOI: 10.1016/j.patrec.2005.08.011.

Han J, Kamber M, Pei J. 2012. Data Mining: Concepts and Techniques. DOI: 10.1016/C2009-061819-5.

He K, Zhang X, Ren S, Sun J. 2016. Deep Residual Learning for Image Recognition. In: 2016 IEEE Conference on Computer Vision and Pattern Recognition (CVPR). IEEE, 770-778. DOI: 10.1109/CVPR.2016.90.

Hothorn T, Lausen B. 2003. Double-bagging: Combining classifiers by bootstrap aggregation. Pattern Recognition 36:1303-1309. DOI: 10.1016/S0031-3203(02)00169-3.

Huang G, Liu Z, Van Der Maaten L, Weinberger KQ. 2017. Densely connected convolutional networks. In: Proceedings - 30th IEEE Conference on Computer Vision and Pattern Recognition, CVPR 2017. DOI: 10.1109/CVPR.2017.243.

Ju C, Bibaut A, van der Laan M. 2018. The relative performance of ensemble methods with deep convolutional neural networks for image classification. Journal of Applied Statistics. DOI: 10.1080/02664763.2018.1441383.

Keras. Available at https://keras.io/

Khan HA, Al Helal A, Ahmed KI. 2014. Handwritten Bangla digit recognition using Sparse Representation Classifier. In: 2014 International Conference on Informatics, Electronics and Vision, ICIEV 2014. DOI: 10.1109/ICIEV.2014.6850817.

Krizhevsky A, Sutskever I, Hinton GE. 2017. ImageNet classification with deep convolutional neural networks. Communications of the ACM. DOI: 10.1145/3065386.

Larsson G, Maire M, Shakhnarovich G. 2019. FractalNet: Ultra-deep neural networks without residuals. In: 5th International Conference on Learning Representations, ICLR 2017 Conference Track Proceedings.

Lin M, Chen Q, Yan S. 2014. Network in network. In: 2nd International Conference on Learning Representations, ICLR 2014 - Conference Track Proceedings.

Manisha N, Sreenivasa E, K. Y. 2016. Role of Offline Handwritten Character Recognition System in Various Applications. International Journal of Computer Applications. DOI:

PeerJ Comput. Sci. reviewing PDF | (CS-2021:01:57252:1:1:NEW 27 Apr 2021) 
768

769

770

771

772

773

774

775

776

777

778

779

780

781

782

783

784

785

786

787

788

789

790

791

792

793

794

795

796

797

798

799

800

801

802

803

804

805

806

807

808

809

810

811

812

813

10.5120/ijca2016908349.

Manoharan S. 2019. A SMART IMAGE PROCESSING ALGORITHM FOR TEXT RECOGNITION, INFORMATION EXTRACTION AND VOCALIZATION FOR THE VISUALLY CHALLENGED. Journal of Innovative Image Processing. DOI: 10.36548/jiip.2019.1.004.

Mor SS, Solanki S, Gupta S, Dhingra S, Jain M, Saxena R. 2019. Handwritten text recognition: With deep learning and android. International Journal of Engineering and Advanced Technology.

Nicolas-Alonso LF, Corralejo R, Gomez-Pilar J, Álvarez D, Hornero R. 2015. Adaptive Stacked Generalization for Multiclass Motor Imagery-Based Brain Computer Interfaces. IEEE Transactions on Neural Systems and Rehabilitation Engineering 23:702-712. DOI: 10.1109/TNSRE.2015.2398573.

Opitz D, Maclin R. 1999. Popular Ensemble Methods: An Empirical Study. Journal of Artificial Intelligence Research. DOI: 10.1613/jair.614.

Pal U, Wakabayashi T, Kimura F. 2007. Handwritten bangla compound character recognition using gradient feature. In: Proceedings - 10th International Conference on Information Technology, ICIT 2007. DOI: 10.1109/ICOIT.2007.4418297.

Peng Y, Yin H. 2017. Markov random field based convolutional neural networks for image classification. In: Lecture Notes in Computer Science (including subseries Lecture Notes in Artificial Intelligence and Lecture Notes in Bioinformatics). DOI: 10.1007/978-3-31968935-7_42.

Perez L, Wang J. 2017. The Effectiveness of Data Augmentation in Image Classification using Deep Learning.

Pham H, Setlur A, Dingliwal S, Lin TH, Poczos B, Huang K, Li Z, Lim J, McCormack C, Vu T. 2020. Robust Handwriting Recognition with Limited and Noisy Data. In: Proceedings of International Conference on Frontiers in Handwriting Recognition, ICFHR. DOI: 10.1109/ICFHR2020.2020.00062.

Purkaystha B, Datta T, Islam MS. 2017. Bengali Handwritten Character Recognition Using Deep Convolutional Neural Network. In: 22-24. DOI: 10.1109/ICCITECHN.2017.8281853.

Rabby AKMSA, Haque S, Islam MS, Abujar S, Hossain SA. 2019. Ekush: A Multipurpose and Multitype Comprehensive Database for Online Off-Line Bangla Handwritten Characters. In: Communications in Computer and Information Science. DOI: 10.1007/978-981-139187-3_14.

Rahaman Mamun M, Al Nazi Z, Salah Uddin Yusuf M. 2018. Bangla Handwritten Digit Recognition Approach with an Ensemble of Deep Residual Networks. 2018 International Conference on Bangla Speech and Language Processing, ICBSLP 2018:21-22. DOI: 10.1109/ICBSLP.2018.8554674.

Rahman MM, Akhand MAH, Islam S, Chandra Shill P, Hafizur Rahman MM. 2015. Bangla Handwritten Character Recognition using Convolutional Neural Network. International Journal of Image, Graphics and Signal Processing 7:42-49. DOI: 10.5815/ijigsp.2015.08.05.

Rajaraman S, Candemir S, Xue Z, Alderson PO, Kohli M, Abuya J, Thoma GR, Antani S. 2018. A novel stacked generalization of models for improved TB detection in chest radiographs. In: Proceedings of the Annual International Conference of the IEEE Engineering in Medicine and Biology Society, EMBS. DOI: 10.1109/EMBC.2018.8512337.

Ranzato MA, Poultney C, Chopra S, LeCun Y. 2007. Efficient learning of sparse representations

PeerJ Comput. Sci. reviewing PDF | (CS-2021:01:57252:1:1:NEW 27 Apr 2021) 
814

815

816

817

818

819

820

821

822

823

824

825

826

827

828

829

830

831

832

833

834

835

836

837

838

839

840

841

842

843

844

845

846

847

848

849

850

851

852

853

854

855

856

857

858

with an energy-based model. In: Advances in Neural Information Processing Systems. DOI: 10.7551/mitpress/7503.003.0147.

Reza S, Amin OB, Hashem MMA. 2020. Basic to Compound: A Novel Transfer Learning Approach for Bengali Handwritten Character Recognition. DOI:

10.1109/icbslp47725.2019.201522.

Sarkar R, Das N, Basu S, Kundu M, Nasipuri M, Basu DK. 2012. CMATERdb1: A database of unconstrained handwritten Bangla and Bangla-English mixed script document image. International Journal on Document Analysis and Recognition. DOI: 10.1007/s10032-0110148-6.

Sarkhel R, Saha AK, Das N. 2015. An enhanced harmony search method for Bangla handwritten character recognition using region sampling. 2015 IEEE 2nd International Conference on Recent Trends in Information Systems, ReTIS 2015 - Proceedings:325-330. DOI: 10.1109/ReTIS.2015.7232899.

Sazal MMR, Biswas SK, Amin MF, Murase K. 2013. Bangla handwritten character recognition using deep belief network. In: 2013 International Conference on Electrical Information and Communication Technology, EICT 2013. DOI: 10.1109/EICT.2014.6777907.

scikit-learn. Available at https://scikit-learn.org/

Sharif SMA, Mohammed N, Mansoor N, Momen S. 2017. A hybrid deep model with HOG features for Bangla handwritten numeral classification. Proceedings of 9th International Conference on Electrical and Computer Engineering, ICECE 2016:463-466. DOI: 10.1109/ICECE.2016.7853957.

Shibly MMA, Tisha TA, Ripon SH. 2021. Stacked Generalization Ensemble Method to Classify Bangla Handwritten Character. In: Shakya S et al. ed. Proceedings of International Conference on Sustainable Expert Systems, Lecture Notes in Networks and Systems 176. Kirtipur, Nepal: Springer Nature Singapore,. DOI: 10.1007/978-981-33-4355-9_46.

Shopon M, Mohammed N, Abedin MA. 2017. Bangla handwritten digit recognition using autoencoder and deep convolutional neural network. In: IWCI 2016 - 2016 International Workshop on Computational Intelligence. DOI: 10.1109/IWCI.2016.7860340.

Simonyan K, Zisserman A. 2015. Very deep convolutional networks for large-scale image recognition. In: 3rd International Conference on Learning Representations, ICLR 2015 Conference Track Proceedings.

Sitaula C, Basnet A, Aryal S. 2021. Vector representation based on a supervised codebook for Nepali documents classi fi cation. :1-18. DOI: $10.7717 /$ peerj-cs.412.

Springenberg JT, Dosovitskiy A, Brox T, Riedmiller M. 2015. Striving for simplicity: The all convolutional net. In: 3rd International Conference on Learning Representations, ICLR 2015 - Workshop Track Proceedings.

Szegedy C, Liu W, Jia Y, Sermanet P, Reed S, Anguelov D, Erhan D, Vanhoucke V, Rabinovich A. 2015. Going deeper with convolutions. In: Proceedings of the IEEE Computer Society Conference on Computer Vision and Pattern Recognition. DOI: 10.1109/CVPR.2015.7298594.

TensorFlow. Available at https://www.tensorflow.org/

Tsai C-F. 2005. Training support vector machines based on stacked generalization for image classification. Neurocomputing 64:497-503. DOI: 10.1016/j.neucom.2004.08.005.

Vinet L, Zhedanov A. 2010. A “missing” family of classical orthogonal polynomials. Math. Intell. DOI: 10.1088/1751-8113/44/8/085201. 
Figure 1

Complexity comparison of Bangla handwritten character with handwritten characters from other languages

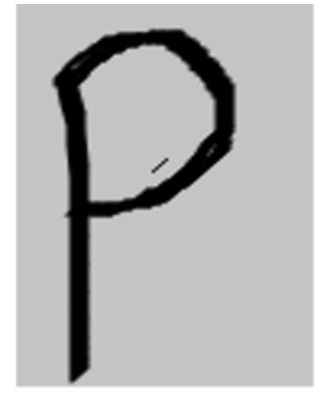

(a) An English handwritten character

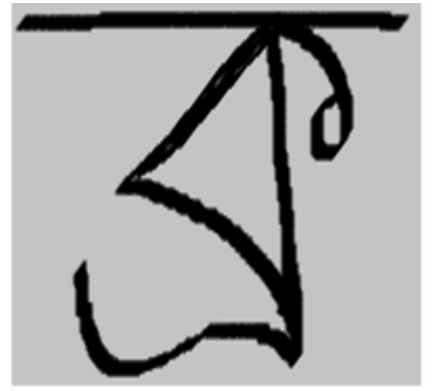

(b) A Bangla handwritten character

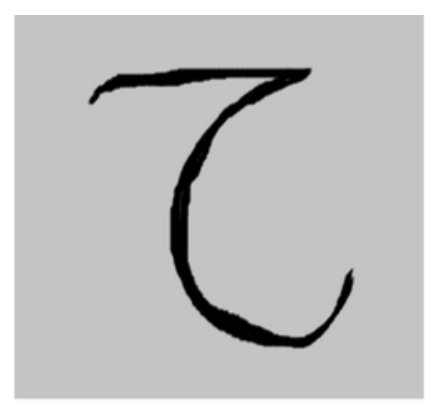

(c) An Arabic handwritten character 
Figure 2

Overview of the workflow of the study

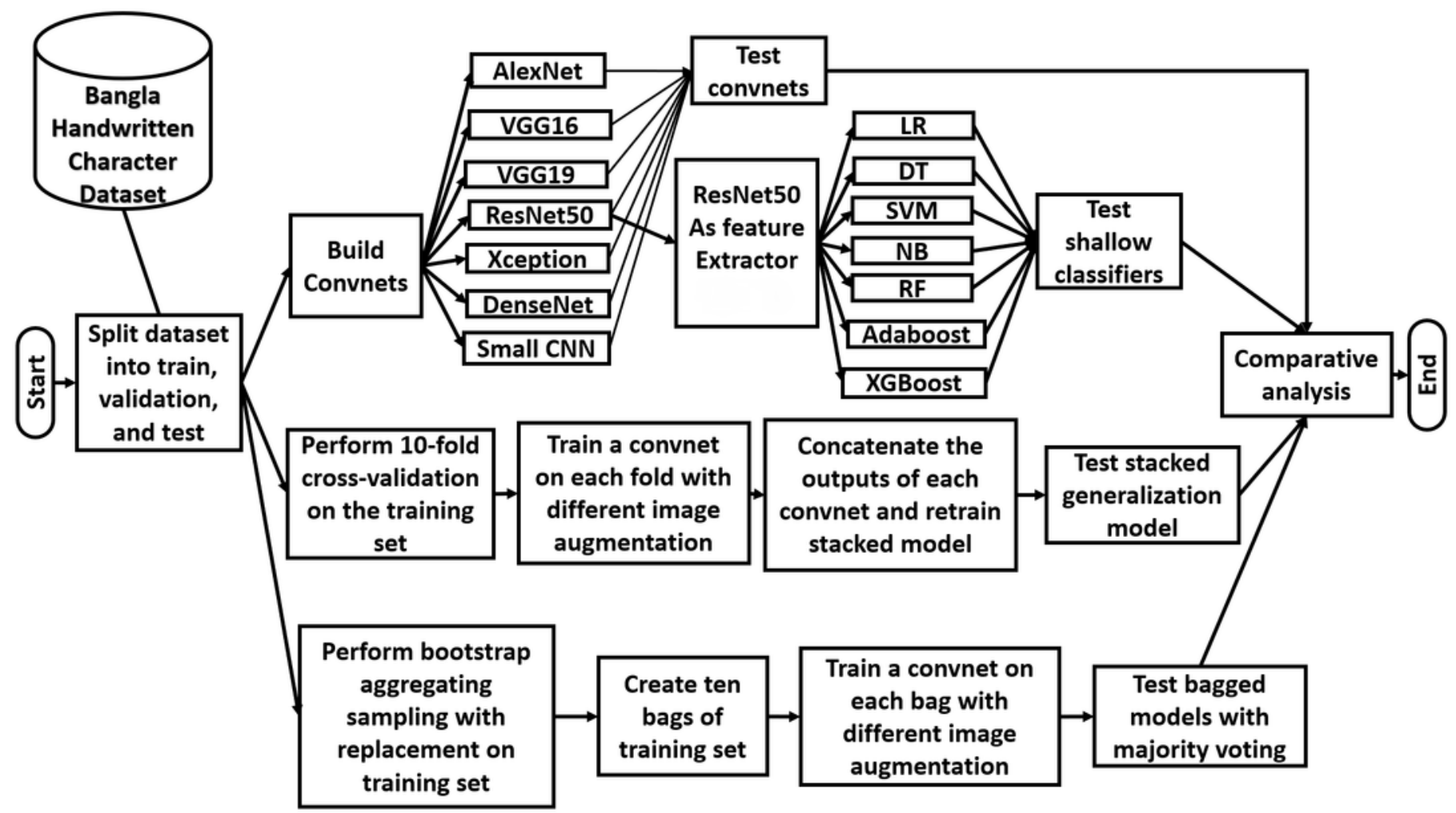


Figure 3

Two building blocks of ResNet architecture

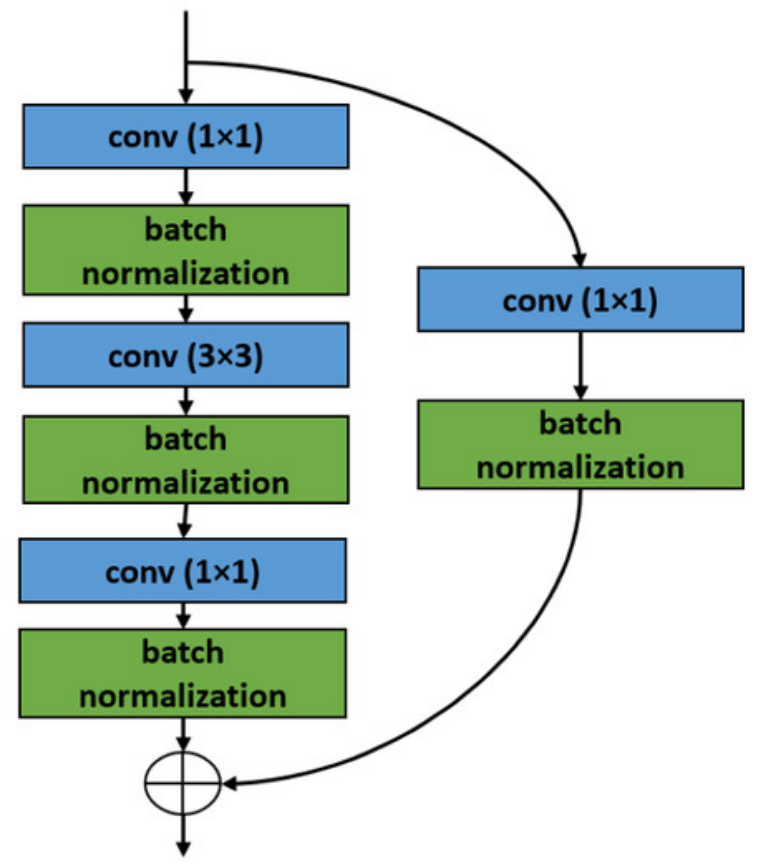

(a) Convolutional block

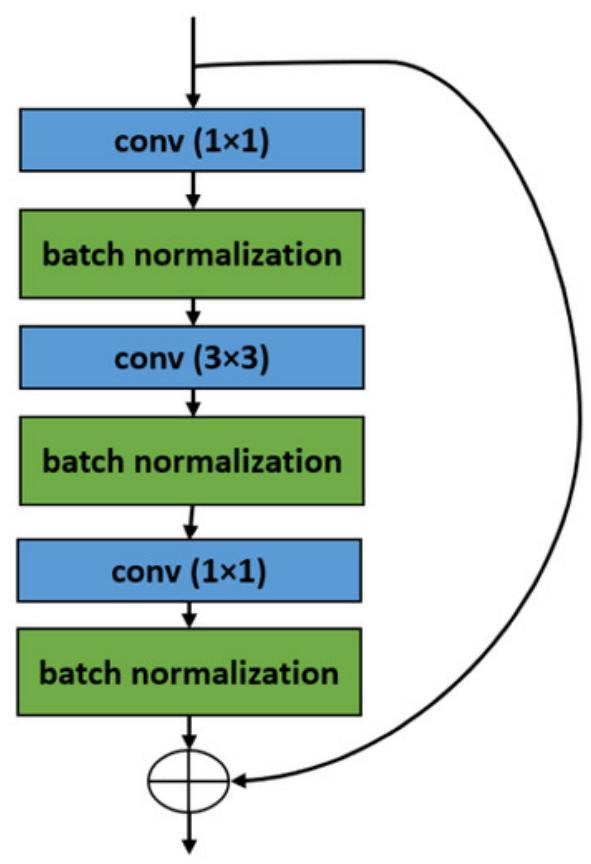

(b) Identity block 
Figure 4

\section{Xception architecture details}

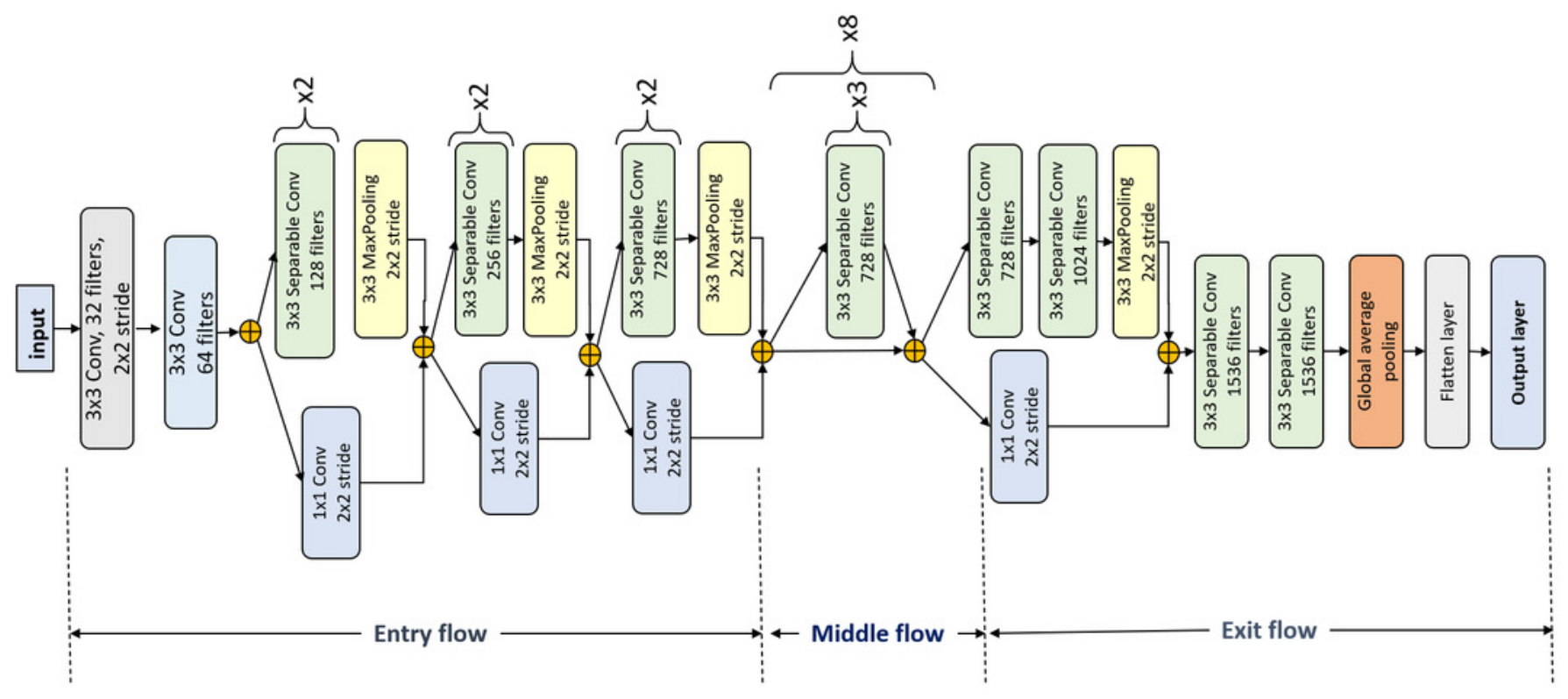


Figure 5

Overview of DenseNet architecture

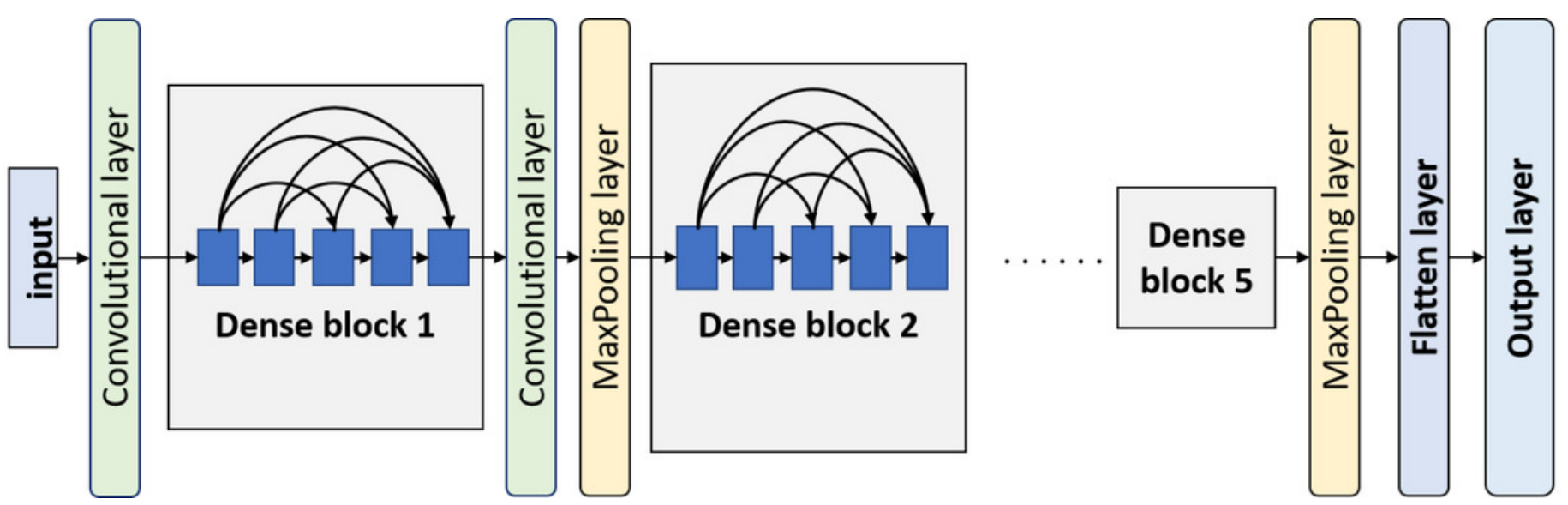


Figure 6

The working procedure of stacked generalization ensemble method

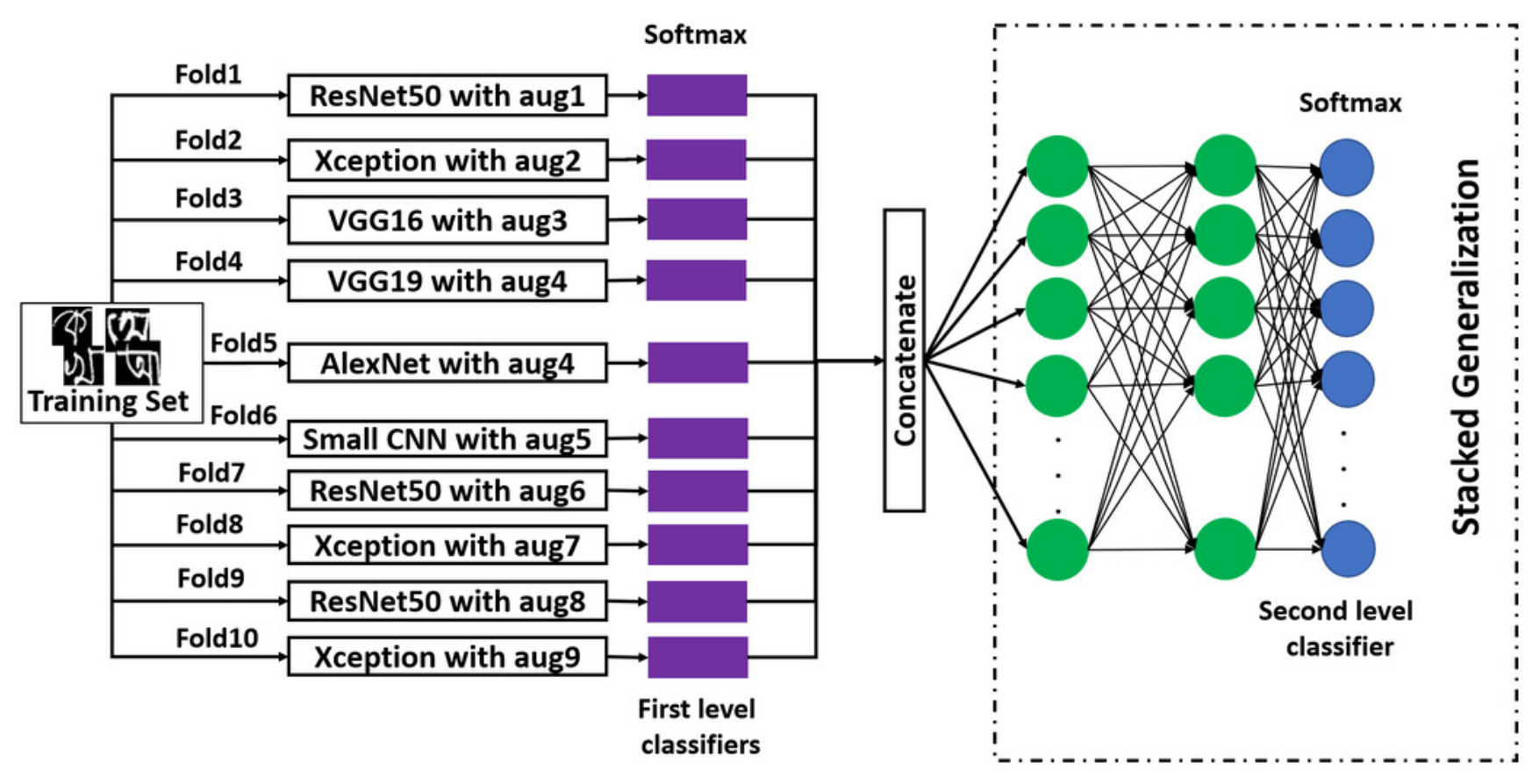


Figure 7

The working procedure of bootstrap aggregating ensemble method

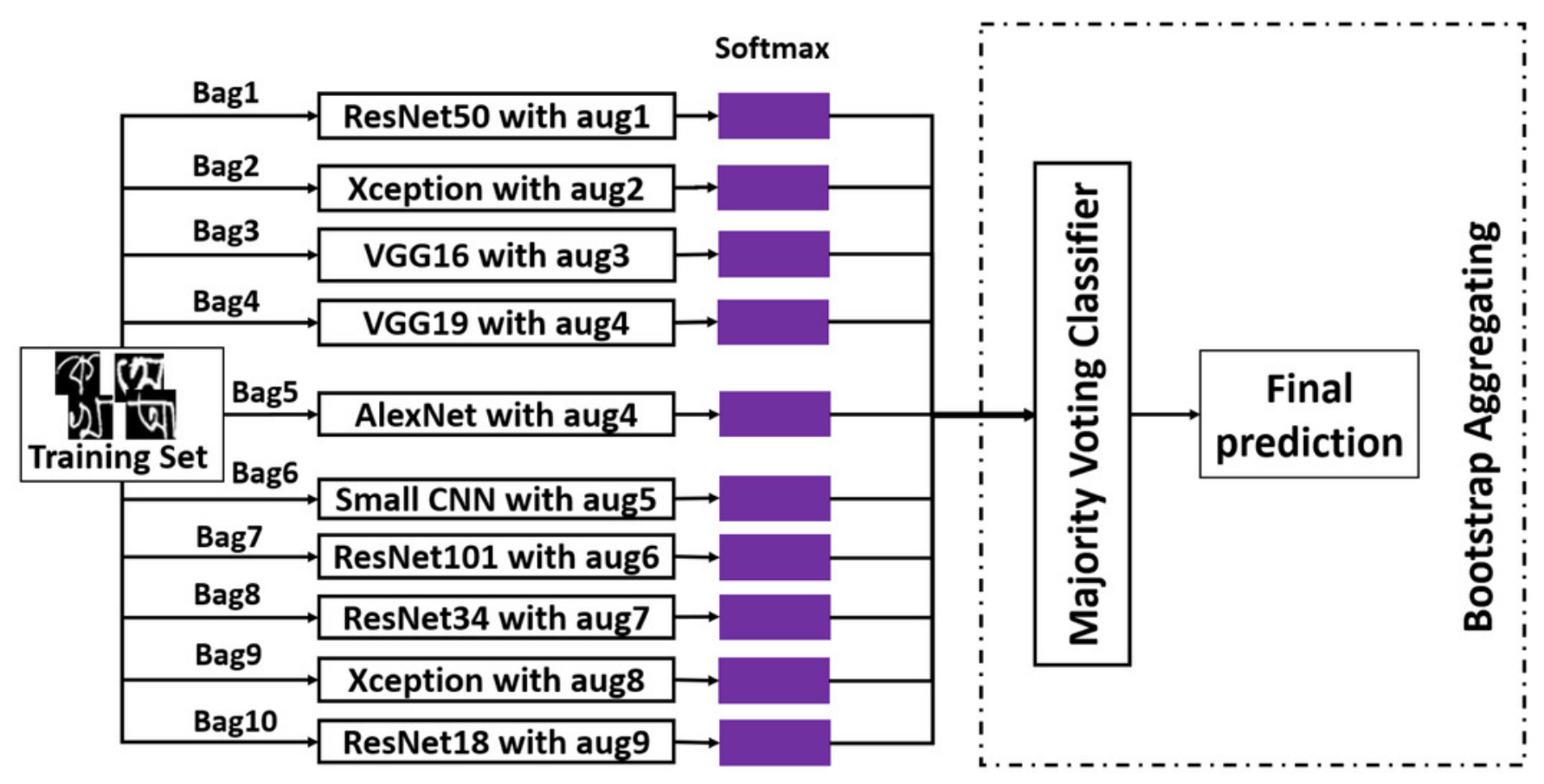




\section{Figure 8}

Few representative images of each character type from the Ekush dataset

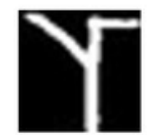

Label: 0

Character: I

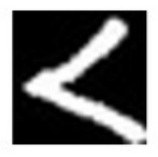

Label: 5

Character:

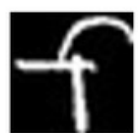

Label: 1 Character:

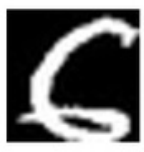

Label: 6 Character: 6

(a) Few modifiers

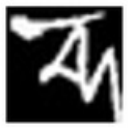

Label: 60

Char: ব্দ

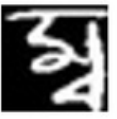

Label: 71

Char: স্ব

(d) Few compounds

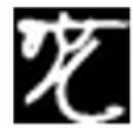

Label: 61

Char: יㅏ

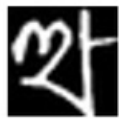

Label: 72

Char: ข्थ

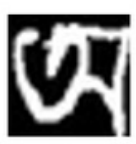

Label: 10

Character: অ

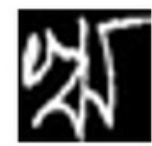

Label: 16

Character: ঋ

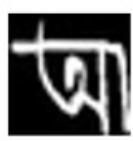

Label: 11

Character: आ

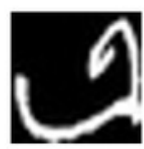

Label: 17

Character: এ

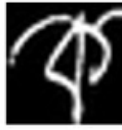

Label: 21

Character: ক

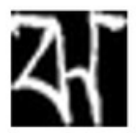

Label: 29

Character: ঝ

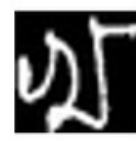

Label: 22

Character: খ

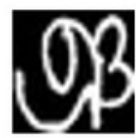

Label: 30 Character: @ (b) Few vowels

(c) Few consonants

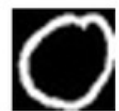

Label: 112

Character: 0

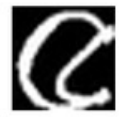

Label: 117

Character: $৫$

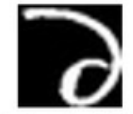

Label: 113

Character: s

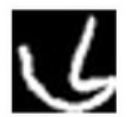

Label: 118

Character: ৬

(e) Few digits 
Figure 9

Validation accuracy vs epochs comparison for the convnets on Ekush dataset

Validation accuracy vs epochs comparison

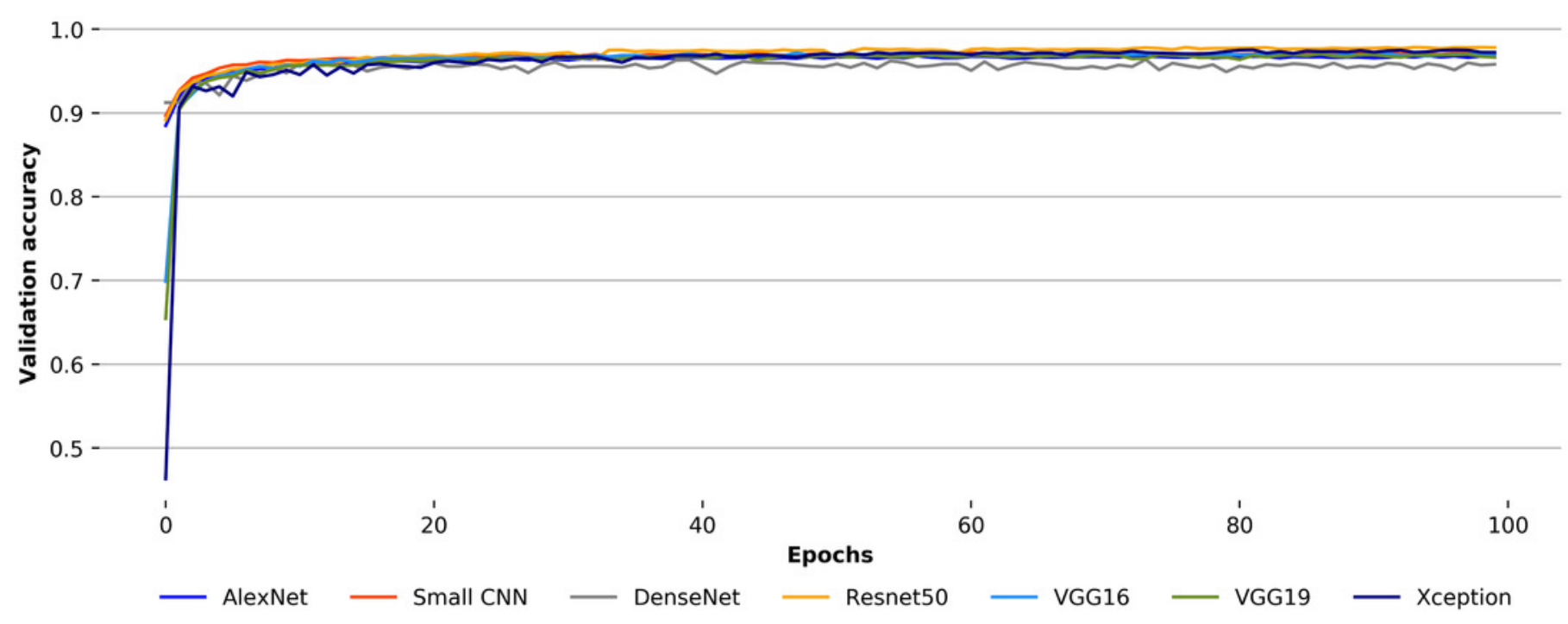


Figure 10

Validation loss vs epochs comparison for the convnets on Ekush dataset

Validation loss vs epochs comparison

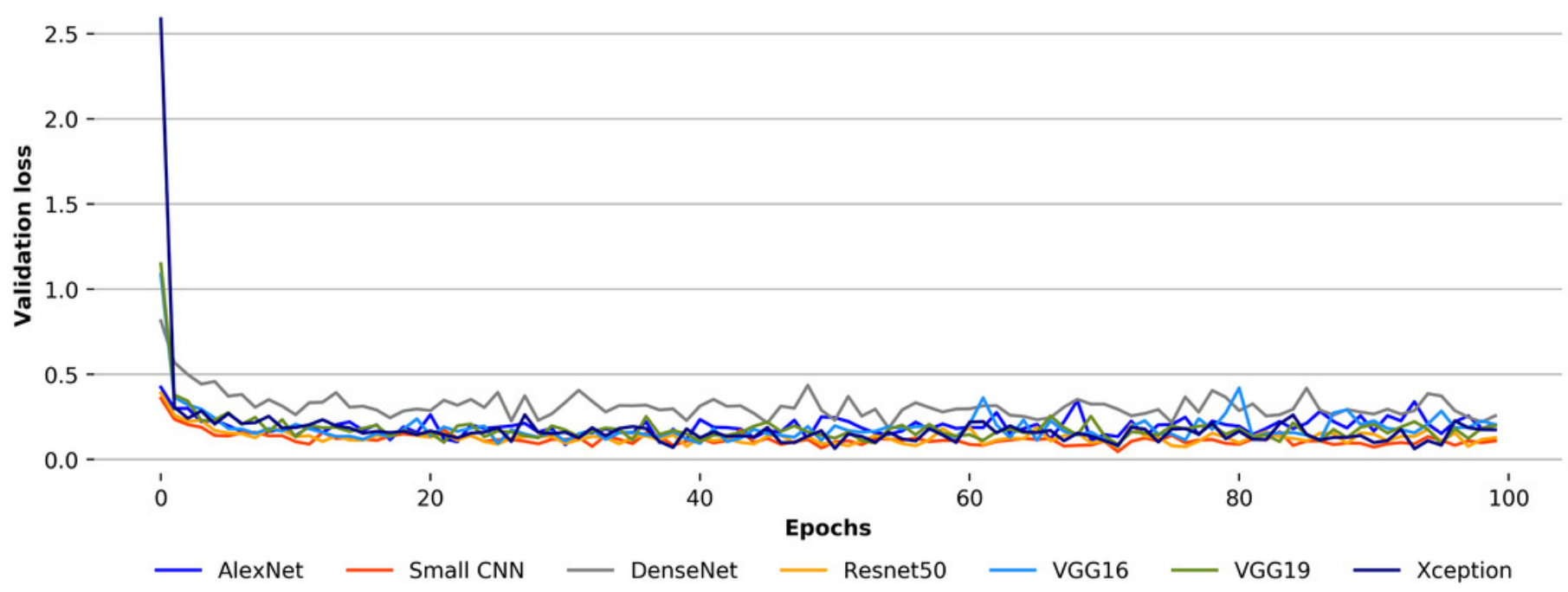




\section{Figure 11}

First and second level classifiers' performances on Ekush dataset

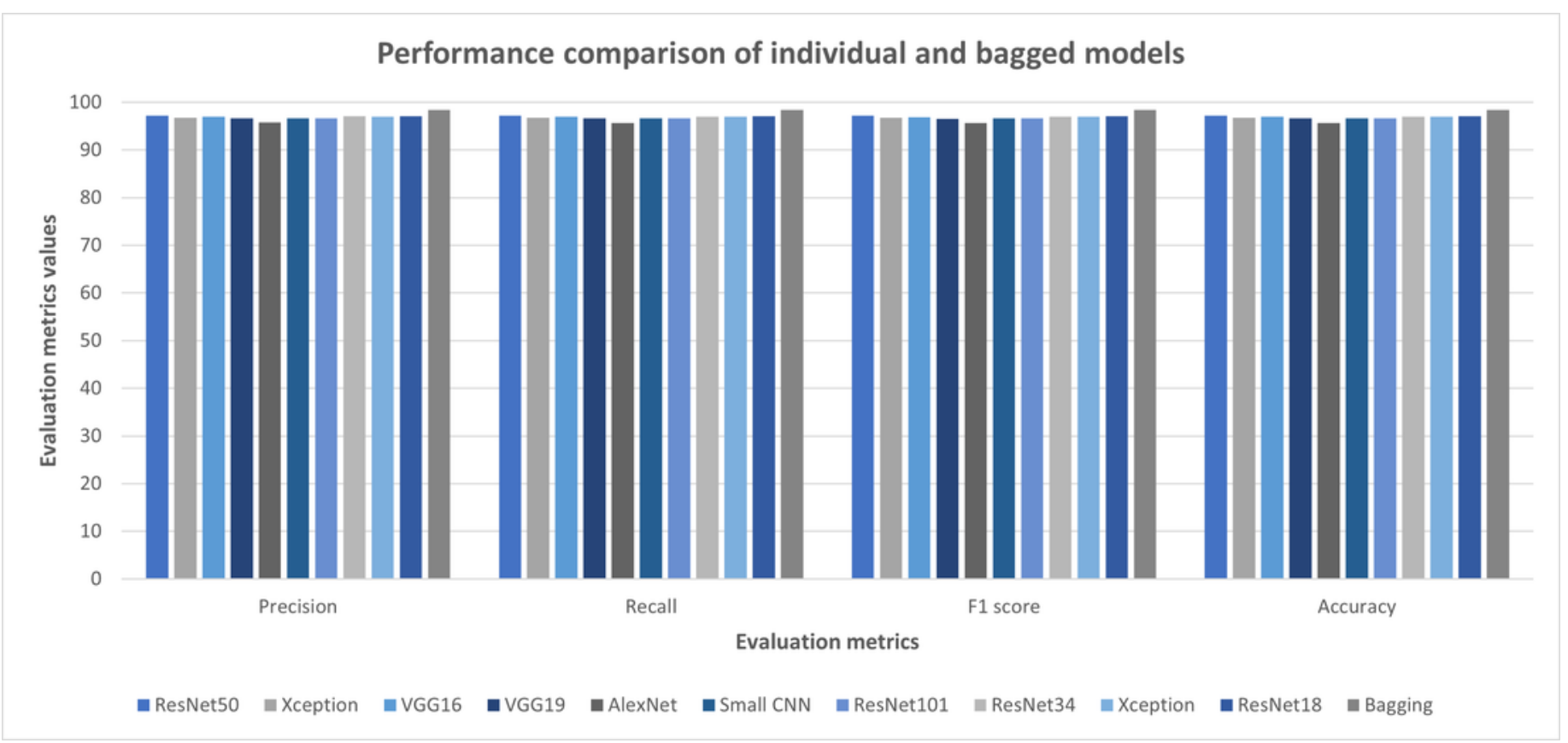




\section{Figure 12}

Individual and bagged classifiers' performances on Ekush dataset

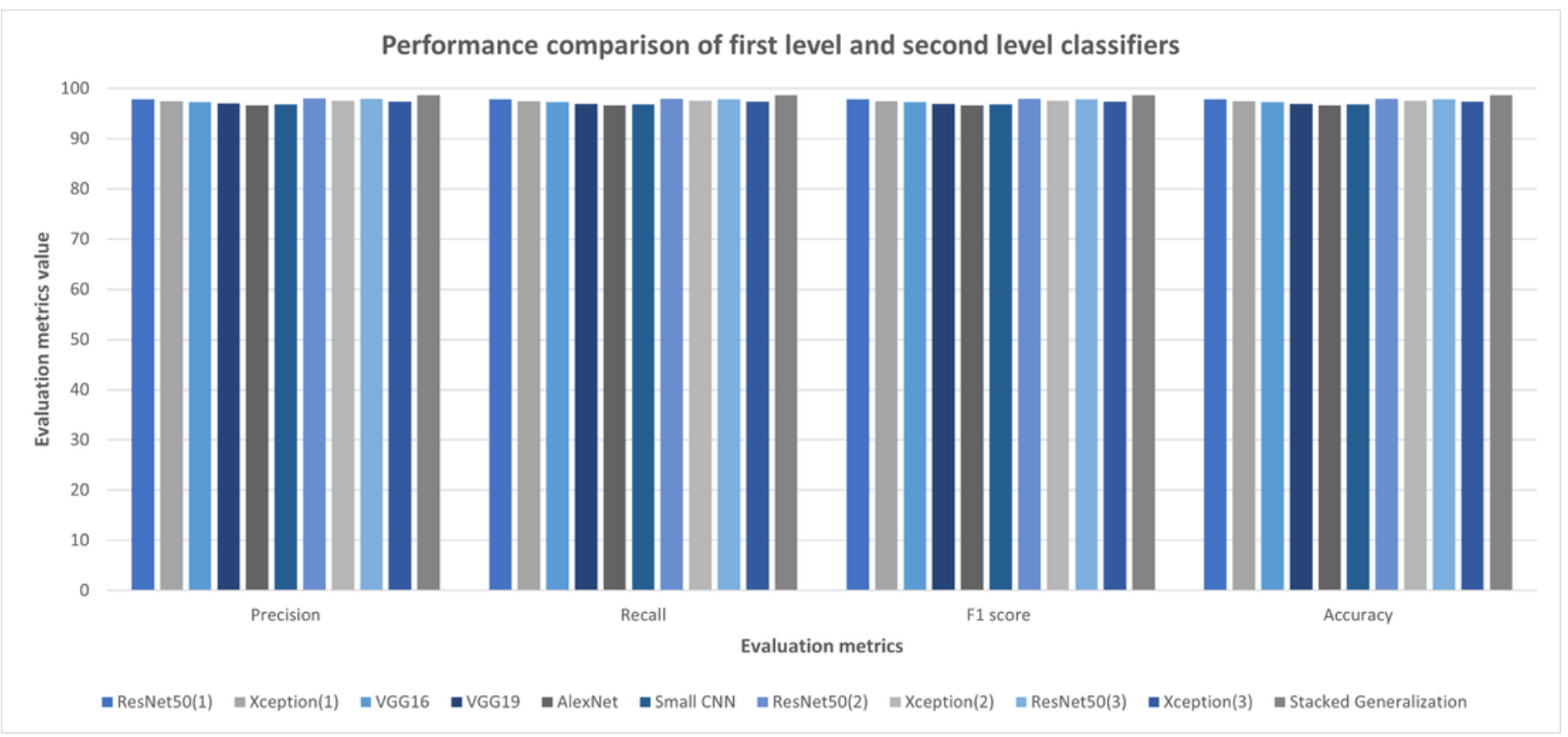


Figure 13

Few misclassified instances from Ekush dataset by ResNet50 model
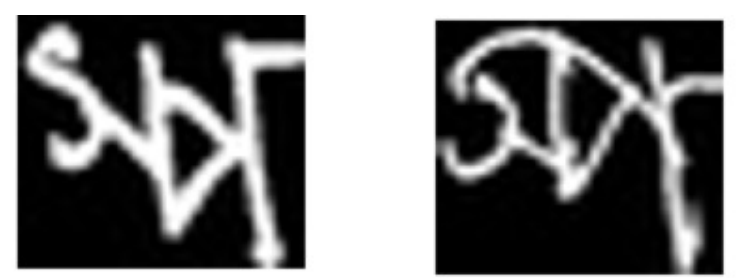

Label: 111

Character: স্्ি
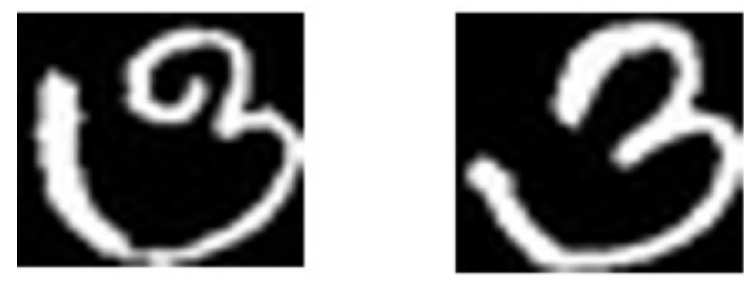

Label: 19

Character: 3
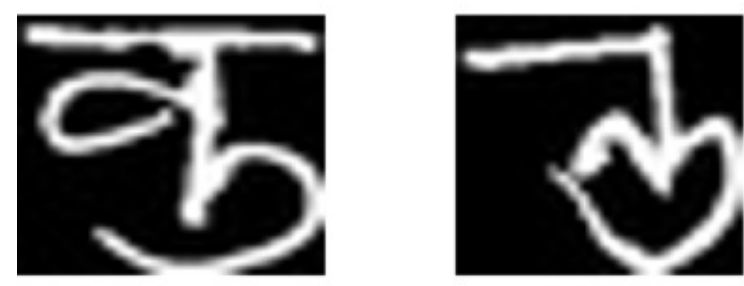

Label: 97

Character: न्ড
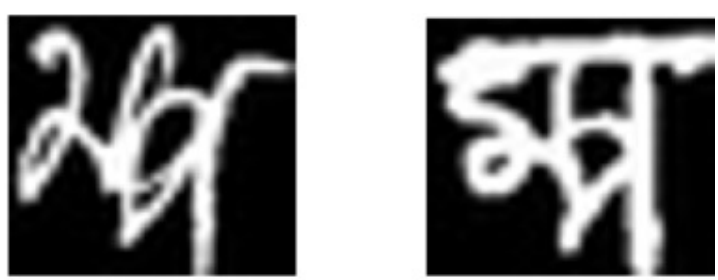

Label: 69

Character: ম্्भ
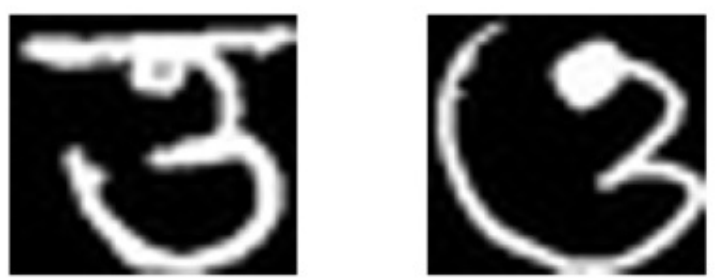

Label: 84

Character: $\overline{3}$
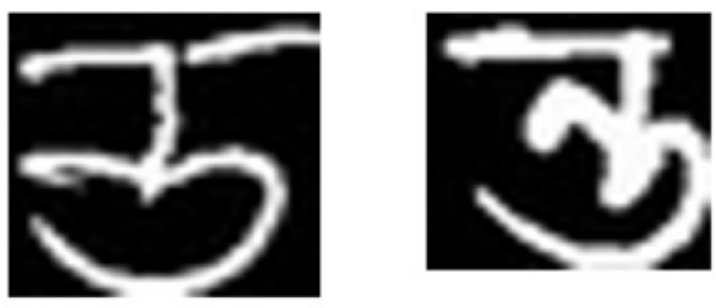

Label: 87

Character: ন্ত 


\section{Table $\mathbf{1}$ (on next page)}

Details of AlexNet, VGG16, VGG19, and a small CNN architectures

The layers descriptions are given for each architecture. For convolutional layer (Conv2D), the dimension of the filter at a convolutional layer e.g., $3 \times 3$ and number of filters e.g., 96 are given. For max-pooling, the pool size e.g., $2 \times 2$ is mentioned. 


\begin{tabular}{|c|c|c|c|}
\hline AlexNet & VGG16 & VGG19 & Small CNN \\
\hline$\left[\begin{array}{l}\text { Conv2D, } 3 \times 3,96 \\
\text { MaxPooling, } 2 \times 2 \\
\text { Conv2D, } 3 \times 3,128 \\
\text { Conv2D, } 3 \times 3,192 \\
\text { Conv2D, } 3 \times 3,256 \\
\text { Conv2D, } 3 \times 3,128 \\
\text { MaxPooling, } 2 \times 2\end{array}\right]$ & $\begin{array}{l}\text { Conv2D, } 3 \times 3,32 \\
\text { Conv2D, } 3 \times 3,32 \\
\text { MaxPooling, } 2 \times 2 \\
\text { Conv2D, } 3 \times 3,64 \\
\text { Conv2D, } 3 \times 3,64 \\
\text { MaxPooling, } 2 \times 2 \\
\text { Conv2D, } 3 \times 3,128 \\
\text { Conv2D, } 3 \times 3,128 \\
\text { Conv2D, } 3 \times 3,128 \\
\text { MaxPooling, } 2 \times 2 \\
\text { Conv2D, } 3 \times 3,175 \\
\text { Conv2D, } 3 \times 3,175 \\
\text { Conv2D, } 3 \times 3,175 \\
\text { MaxPooling, 2 } \times 2 \\
\text { Conv2D, } 3 \times 3,225 \\
\text { Conv2D, } 3 \times 3,225 \\
\text { Conv2D, } 3 \times 3,225 \\
\text { MaxPooling, } 1 \times 1 \\
\end{array}$ & $\begin{array}{l}\text { Conv2D, } 3 \times 3,32 \\
\text { Conv2D, } 3 \times 3,32 \\
\text { MaxPooling, } 2 \times 2 \\
\text { Conv2D, } 3 \times 3,64 \\
\text { Conv2D, } 3 \times 3,64 \\
\text { MaxPooling, } 2 \times 2 \\
\text { Conv2D, } 3 \times 3,128 \\
\text { Conv2D, } 3 \times 3,128 \\
\text { Conv2D, } 3 \times 3,128 \\
\text { Conv2D, } 3 \times 3,128 \\
\text { MaxPooling, } 2 \times 2 \\
\text { Conv2D, } 3 \times 3,192 \\
\text { Conv2D, } 3 \times 3,192 \\
\text { Conv2D, } 3 \times 3,192 \\
\text { Conv2D, } 3 \times 3,192 \\
\text { MaxPooling, } 2 \times 2 \\
\text { Conv2D, } 3 \times 3,256 \\
\text { Conv2D, } 3 \times 3,256 \\
\text { Conv2D, } 3 \times 3,256 \\
\text { Conv2D, } 3 \times 3,256 \\
\text { MaxPooling, } 2 \times 2 \\
\end{array}$ & $\left|\begin{array}{c}\text { Conv2D, } 3 \times 3,50 \\
\text { Conv2D, } 3 \times 3,75 \\
\text { MaxPooling, } 2 \times 2 \\
\text { Conv2D, } 3 \times 3,125 \\
\text { MaxPooling, } 2 \times 2 \\
\text { Conv2D, } 3 \times 3,175 \\
\text { MaxPooling, } 2 \times 2 \\
\text { Conv2D, } 3 \times 3,225 \\
\text { MaxPooling, } 2 \times 2\end{array}\right|$ \\
\hline Flatten & Flatten & Flatten & Flatten \\
\hline $1^{\text {st }}$ Dense layer & $1^{\text {st }}$ Dense layer & $1^{\text {st }}$ Dense layer & $\begin{array}{c}1^{\text {st }} \text { Dense layer }+ \\
\text { Dropout }(50 \%)\end{array}$ \\
\hline $2^{\text {nd }}$ Dense layer & $2^{\text {nd }}$ Dense layer & $2^{\text {nd }}$ Dense layer & $\begin{array}{c}2^{\text {nd }} \text { Dense layer }+ \\
\text { Dropout }(30 \%\end{array}$ \\
\hline Softmax output layer & Softmax output layer & Softmax output layer & Softmax output layer \\
\hline
\end{tabular}

1 


\section{Table 2 (on next page)}

Different image augmentation hyperparameters 


\begin{tabular}{|c|c|}
\hline Name & Augmentation hyperparameters \\
\hline aug0 & no image augmentation \\
\hline aug1 & $A_{1}=10 \wedge A_{2}=0.1 \wedge A_{3}=0.1 \wedge A_{4}=0.1$ \\
\hline aug2 & $A_{1}=9 \wedge A_{2}=0.09 \wedge A_{3}=0.09 \wedge A_{4}=0.09$ \\
\hline aug3 & $A_{1}=11 \wedge A_{2}=0.11 \wedge A_{3}=0.11 \wedge A_{4}=0.11$ \\
\hline aug4 & $A_{1}=10$ \\
\hline aug5 & $A_{1}=15 \wedge A_{2}=0.15 \wedge A_{3}=0.15 \wedge A_{4}=0.15$ \\
\hline aug6 & $A_{1}=11 \wedge A_{2}=0.11 \wedge A_{3}=0.11 \wedge A_{4}=0.10$ \\
\hline aug7 & $A_{1}=13 \wedge A_{2}=0.09 \wedge A_{3}=0.1 \wedge A_{4}=0.11$ \\
\hline aug8 & $A_{1}=14 \wedge A_{2}=0.1 \wedge A_{3}=0.1 \wedge A_{4}=0.1$ \\
\hline aug9 & $A_{1}=15 \wedge A_{2}=0.11 \wedge A_{3}=0.11 \wedge A_{4}=0.11$ \\
\hline
\end{tabular}




\section{Table 3(on next page)}

Ekush dataset details 


\begin{tabular}{|l|l|l|}
\hline Character Type & No. of Classes & No. of Instances \\
\hline Modifier & 10 & 54,829 \\
\hline Basic Character & 50 & 307,316 \\
\hline Compound Character & 52 & 306,231 \\
\hline Digit & 10 & 61,374 \\
\hline Total & 122 & 729,750 \\
\hline
\end{tabular}




\section{Table 4 (on next page)}

Performance comparison with state-of-the-art works on Bangla handwritten character recognition 


\begin{tabular}{|l|l|l|l|l|}
\hline Work & Dataset & $\begin{array}{l}\text { Number of } \\
\text { characters }\end{array}$ & Method & $\begin{array}{l}\text { Accuracy } \\
\text { (\%) }\end{array}$ \\
\hline $\begin{array}{l}\text { (Pal, Wakabayashi \& Kimura, } \\
\text { 2007) }\end{array}$ & $\begin{array}{l}\text { Own collected } \\
\text { dataset. }\end{array}$ & 138 & MQDF & 85.90 \\
\hline Rahman et al., 2015) & $\begin{array}{l}\text { Own prepared } \\
\text { dataset with 20000 } \\
\text { samples }\end{array}$ & 50 & Convnets & 85.96 \\
\hline $\begin{array}{l}\text { (Purkaystha, Datta \& Islam, } \\
\text { 2017) }\end{array}$ & $\begin{array}{l}\text { BanglaLekha } \\
\text { Isolated (Biswas et } \\
\text { al., 2017) }\end{array}$ & 80 & Convnets & 88.93 \\
\hline (Bhattacharya et al., 2016) & $\begin{array}{l}\text { Own collected } \\
\text { dataset. }\end{array}$ & 152 & SVM & 94.3 \\
\hline (Alif, Ahmed \& Hasan, 2018) & $\begin{array}{l}\text { CMATERdb (Sarkar } \\
\text { et al., 2012) }\end{array}$ & 73 & $\begin{array}{l}\text { Modified } \\
\text { ResNet18 }\end{array}$ & 95.10 \\
\hline $\begin{array}{l}\text { Rahaman Mamun, Al Nazi \& } \\
\text { Salah Uddin Yusuf, 2018) }\end{array}$ & $\begin{array}{l}\text { NumtaDB (Alam et } \\
\text { al., 2018) }\end{array}$ & 10 & Ensemble & 96.69 \\
\hline (Azad Rabby et al., 2018) & $\begin{array}{l}\text { Ekush (Rabby et al., } \\
\text { 2019) }\end{array}$ & 122 & Convnets & 97.73 \\
\hline Proposed method & $\begin{array}{l}\text { Ekush (Rabby et al., } \\
\text { 2019) }\end{array}$ & $\mathbf{1 2 2}$ & $\begin{array}{l}\text { Stacked } \\
\text { Generalization }\end{array}$ & $\mathbf{9 8 . 6 8}$ \\
\hline Proposed method & $\begin{array}{l}\text { Ekush (Rabby et al., } \\
\text { 2019) }\end{array}$ & $\mathbf{1 2 2}$ & Bagging & $\mathbf{9 8 . 3 7}$ \\
\hline Proposed method & $\begin{array}{l}\text { BanglaLekha } \\
\text { Isolated (Biswas et } \\
\text { al., 2017) }\end{array}$ & $\mathbf{8 4}$ & $\begin{array}{l}\text { Stacked } \\
\text { Generalization }\end{array}$ & $\mathbf{9 2 . 6 7}$ \\
\hline Proposed method & $\begin{array}{l}\text { BanglaLekha } \\
\text { Isolated (Biswas et } \\
\text { al., 2017) }\end{array}$ & $\mathbf{8 4}$ & Bagging & $\mathbf{9 3 . 5 5}$ \\
\hline
\end{tabular}




\section{Table 5 (on next page)}

All models' performances on Ekush and BanglaLekha-Isolated datasets 


\begin{tabular}{|c|c|c|c|c|c|c|}
\hline Dataset & Methods & Models & $\begin{array}{l}\text { Precision } \\
(\%)\end{array}$ & Recall (\%) & $\begin{array}{l}\text { F1-score } \\
(\%)\end{array}$ & $\begin{array}{l}\text { Accuracy } \\
(\%)\end{array}$ \\
\hline \multirow{16}{*}{$\begin{array}{l}\text { Ekush } \\
\text { Dataset }\end{array}$} & \multirow[t]{7}{*}{ Convnets } & AlexNet & 96.80 & 96.80 & 96.79 & 96.80 \\
\hline & & VGG16 & 97.06 & 97.05 & 97.04 & 97.05 \\
\hline & & VGG19 & 96.99 & 96.97 & 96.97 & 96.97 \\
\hline & & ResNet50 & 97.82 & 97.81 & 97.81 & 97.81 \\
\hline & & Xception & 97.64 & 97.63 & 97.63 & 97.63 \\
\hline & & DenseNet & 96.31 & 96.25 & 96.26 & 96.25 \\
\hline & & Small CNN & 97.33 & 97.30 & 97.30 & 97.30 \\
\hline & \multirow{4}{*}{$\begin{array}{l}\text { ResNet50 as } \\
\text { the feature } \\
\text { extractor }\end{array}$} & Logistic Regression & 97.76 & 97.76 & 97.76 & 97.76 \\
\hline & & SVM & 97.76 & 97.75 & 97.75 & 97.75 \\
\hline & & Naïve Bayes & 97.2 & 97.15 & 97.16 & 97.15 \\
\hline & & Decision Tree & 95.75 & 95.74 & 95.74 & 95.74 \\
\hline & \multirow[t]{5}{*}{ Ensemble } & Stacked Generalization & 98.69 & 98.68 & 98.68 & 98.68 \\
\hline & & Bootstrap Aggregating & 98.38 & 98.37 & 98.37 & 98.37 \\
\hline & & Adaboost & 96.42 & 96.36 & 96.36 & 96.37 \\
\hline & & Xgboost & 96.59 & 96.58 & 96.58 & 96.58 \\
\hline & & Random Forest & 97.33 & 97.32 & 97.31 & 97.32 \\
\hline \multirow{16}{*}{$\begin{array}{l}\text { BanglaLekha } \\
\text {-Isolated } \\
\text { Dataset }\end{array}$} & \multirow[t]{7}{*}{ Convnets } & AlexNet & 88.99 & 88.88 & 88.89 & 88.88 \\
\hline & & VGG16 & 92.16 & 92.11 & 92.10 & 92.11 \\
\hline & & VGG19 & 89.86 & 89.75 & 89.76 & 89.75 \\
\hline & & ResNet50 & 92.68 & 92.63 & 92.65 & 92.63 \\
\hline & & Xception & 90.37 & 90.19 & 90.22 & 90.19 \\
\hline & & DenseNet & 89.60 & 89.41 & 89.50 & 89.41 \\
\hline & & Small CNN & 92.63 & 92.59 & 92.58 & 92.59 \\
\hline & \multirow{4}{*}{$\begin{array}{l}\text { ResNet50 as } \\
\text { the feature } \\
\text { extractor }\end{array}$} & Logistic Regression & 92.24 & 92.17 & 92.19 & 92.17 \\
\hline & & SVM & 92.09 & 92.03 & 92.04 & 92.03 \\
\hline & & Naïve Bayes & 91.87 & 91.67 & 91.72 & 91.67 \\
\hline & & Decision Tree & 90.12 & 90.04 & 90.06 & 90.04 \\
\hline & \multirow[t]{5}{*}{ Ensemble } & Stacked Generalization & 92.78 & 92.67 & 92.67 & 92.67 \\
\hline & & Bootstrap Aggregating & 93.60 & 93.55 & 93.55 & 93.55 \\
\hline & & Adaboost & 91.72 & 91.62 & 91.66 & 91.62 \\
\hline & & Xgboost & 91.92 & 91.86 & 91.88 & 91.86 \\
\hline & & Random Forest & 92.28 & 92.20 & 92.22 & 92.20 \\
\hline
\end{tabular}




\section{Table 6(on next page)}

Training and testing time of different models on Ekush dataset 
1

\begin{tabular}{|l|l|l|}
\hline Model & $\begin{array}{l}\text { Time to process an image } \\
\text { during training (millisecond) }\end{array}$ & $\begin{array}{l}\text { Time to predict an image } \\
\text { during testing (millisecond) }\end{array}$ \\
\hline AlexNet & 0.9766 & 0.3672 \\
\hline Small CNN & 0.3916 & 0.1582 \\
\hline DenseNet & 1.468 & 0.4307 \\
\hline ResNet50 & 0.4727 & 0.2031 \\
\hline VGG16 & 0.332 & $\mathbf{0 . 1 5 7 2}$ \\
\hline VGG19 & $\mathbf{0 . 3 2 7 1}$ & 0.336 \\
\hline Xception & 0.5469 & 0.1914 \\
\hline
\end{tabular}

2 


\section{Table 7 (on next page)}

False positive, false negative of a few classes of ResNet50 model for Ekush dataset 
1

\begin{tabular}{|c|c|c|c|c|}
\hline Performance & Class & Support & $\begin{array}{c}\text { Number of } \\
\text { false-negative }\end{array}$ & $\begin{array}{c}\text { Number of } \\
\text { false positive }\end{array}$ \\
\hline \multirow{3}{*}{ Poor } & 111 & 156 & 47 & 32 \\
\cline { 2 - 5 } & 46 & 894 & 60 & 60 \\
\cline { 2 - 5 } & 84 & 872 & 63 & 53 \\
\cline { 2 - 5 } & 97 & 642 & 48 & 53 \\
\hline \multirow{2}{*}{ Average } & 103 & 924 & 46 & 2 \\
\cline { 2 - 5 } & 16 & 926 & 15 & 46 \\
\cline { 2 - 5 } & 39 & 926 & 42 & 2 \\
\hline Good & 20 & 928 & 5 & 6 \\
\cline { 2 - 5 } & 96 & 926 & 3 & 46 \\
\hline
\end{tabular}

2 